\title{
Review
}

\section{Quality control of herbal medicines}

\author{
Yi-Zeng Liang ${ }^{\mathrm{a}, *}$, Peishan Xie ${ }^{\mathrm{b}}$, Kelvin Chan ${ }^{\mathrm{c}}$ \\ ${ }^{a}$ Research Center of Modernization of Chinese Medicines, Institute of Chemometrics and Intelligent Analytical Instruments, \\ College of Chemistry and Chemical Engineering, Central South University, Changsha 410083, PR China \\ ${ }^{\mathrm{b}}$ Zhuhai Chromap Institute of Herbal Medicine Research, Zhuhai 519085, PR China \\ ${ }^{\mathrm{c}}$ Research and Development Division, School of Chinese Medicine, Hong Kong Baptist University, Hong Kong, SAR, PR China
}

Received 4 April 2004; accepted 6 August 2004

Available online 23 September 2004

\begin{abstract}
Different chromatographic and electrophoretic techniques commonly used in the instrumental inspection of herbal medicines (HM) are first comprehensively reviewed. Chemical fingerprints obtained by chromatographic and electrophoretic techniques, especially by hyphenated chromatographies, are strongly recommended for the purpose of quality control of herbal medicines, since they might represent appropriately the "chemical integrities" of the herbal medicines and therefore be used for authentication and identification of the herbal products. Based on the conception of phytoequivalence, the chromatographic fingerprints of herbal medicines could be utilized for addressing the problem of quality control of herbal medicines. Several novel chemometric methods for evaluating the fingerprints of herbal products, such as the method based on information theory, similarity estimation, chemical pattern recognition, spectral correlative chromatogram (SCC), multivariate resolution, etc. are discussed in detail with examples, which showed that the combination of chromatographic fingerprints of herbal medicines and the chemometric evaluation might be a powerful tool for quality control of herbal products.
\end{abstract}

(C) 2004 Elsevier B.V. All rights reserved.

Keywords: Herbal medicines; Fingerprints; Quality control; Chemometrics

\section{Contents}

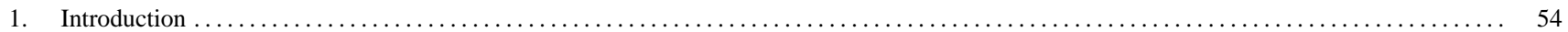

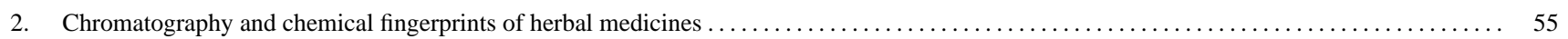

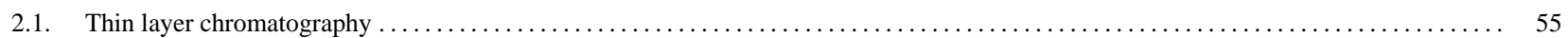

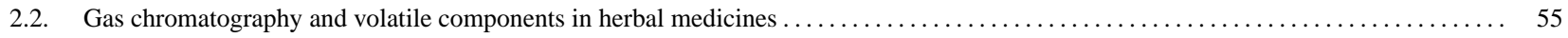

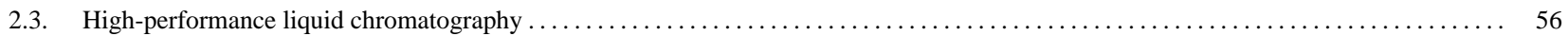

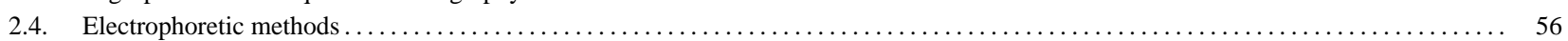

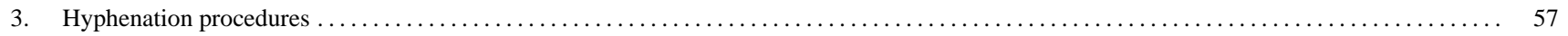

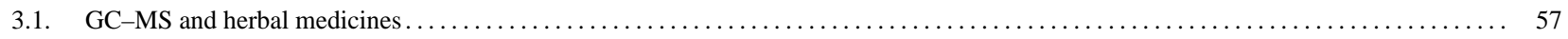

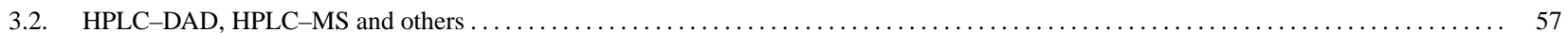

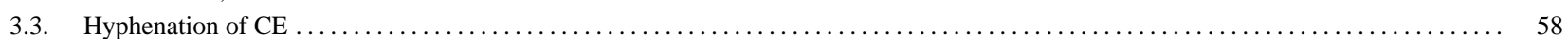

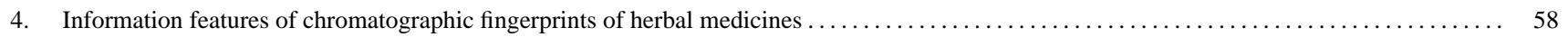

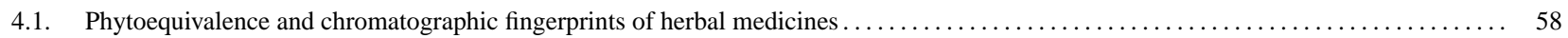

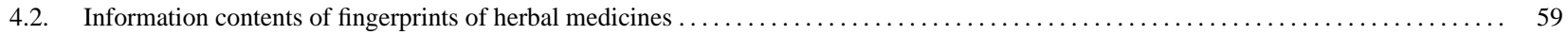

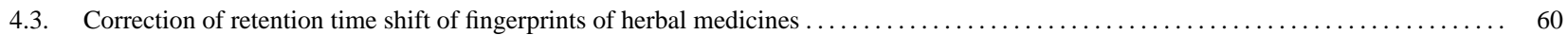

\footnotetext{
* Corresponding author. Tel.: +86 7318822841 ; fax: +867318825637.

E-mail address: yizeng_liang@263.net (Y.-Z. Liang).
} 


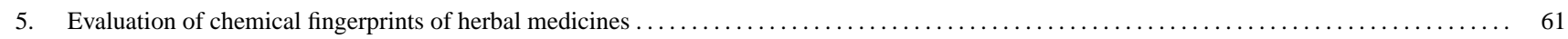

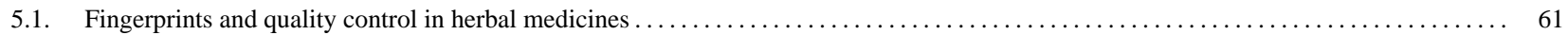

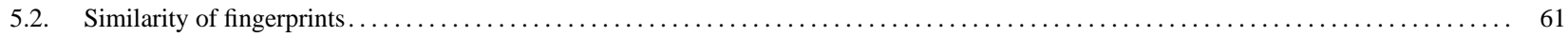

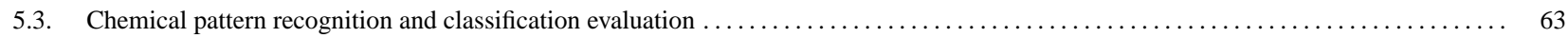

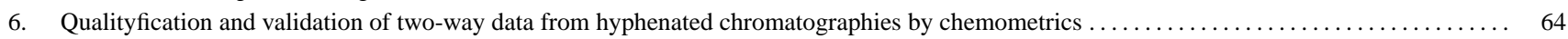

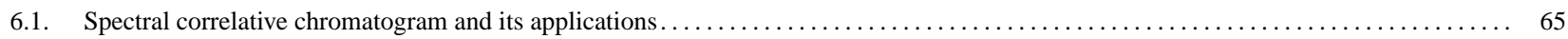

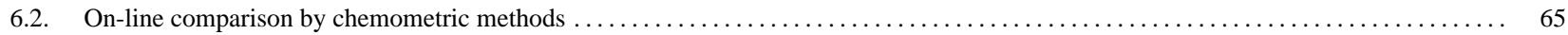

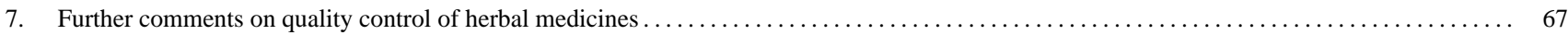

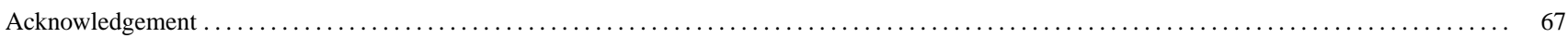

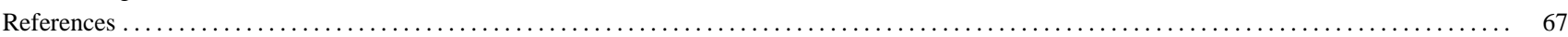

\section{Introduction}

The traditional herbal medicines (HM) and their preparations have been widely used for thousands of years in many oriental countries, such as in China, Korea, Japan, etc. However, one of the characteristics of oriental herbal medicine preparations is that all the herbal medicines, either presenting as single herbs or as collections of herbs in composite formulae, are extracted with boiling water during the decoction process. This may be the main reason why quality control of oriental herbal drugs is more difficult than that of western drug. As pointed in "General Guidelines for Methodologies on Research and Evaluation of Traditional Medicines (World Health Organization, 2000)" [1], "Despite its existence and continued use over many centuries, and its popularity and extensive use during the last decade, traditional medicine has not been officially recognized in most countries. Consequently, education, training and research in this area have not been accorded due attention and support. The quantity and quality of the safety and efficacy data on traditional medicine are far from sufficient to meet the criteria needed to support its use world-wide. The reasons for the lack of research data are due to not only to health care policies, but also to a lack of adequate or accepted research methodology for evaluating traditional medicine".

In general, one or two markers or pharmacologically active components in herbs and or herbal mixtures were currently employed for evaluating the quality and authenticity of herbal medicines, in the identification of the single herb or HM preparations, and in assessing the quantitative herbal composition of an herbal product. This kind of the determination, however, does not give a complete picture of a herbal product, because multiple constituents are usually responsible for its therapeutic effects. These multiple constituents may work 'synergistically' and could hardly be separated into active parts. Moreover, the chemical constituents in component herbs in the HM products may vary depending on harvest seasons, plant origins, drying processes and other factors. Thus, it seems to be necessary to determine most of the phytochemical constituents of herbal products in order to ensure the reliability and repeatability of pharmacological and clinical research, to understand their bioactivities and possible side effects of active compounds and to enhance product quality control [2-4]. Thus, several chromatographic techniques, such as high-performance liquid chromatography (HPLC), gas chromatography (GC), capillary electrophoresis (CE) and thin layer chromatography (TLC), can be applied for this kind of documentation. In this way, the full herbal product could be regarded as the active 'compound'. The concept of phytoequivalence was developed in Germany in order to ensure consistency of herbal products [5]. According to this concept, a chemical profile, such as a chromatographic fingerprint, for a herbal product should be constructed and compared with the profile of a clinically proven reference product.

In 2004, the Chinese State Food and Drug Administration (SFDA) will regulate the compositions of liquid injection with HM ingredients using stringent quality procedures such as chemical assay and standardization. Fingerprints of HM liquid injections are compulsorily carried out for this purpose. In addition, among the various experimental techniques, chromatographic methods are highly recommended for finding out the fingerprints of these products [6-12].

By definition, a chromatographic fingerprint of a HM is, in practice, a chromatographic pattern of the extract of some common chemical components of pharmacologically active and or chemically characteristics $[6-7,11]$. This chromatographic profile should be featured by the fundamental attributions of "integrity" and "fuzziness" or "sameness" and "differences" so as to chemically represent the HM investigated [11,13]. It is suggested that with the help of chromatographic fingerprints obtained, the authentication and identification of herbal medicines can be accurately conducted ("integrity") even if the amount and/or concentration of the chemically characteristic constituents are not exactly the same for different samples of this HM (hence, "fuzziness") or, the chromatographic fingerprints could demonstrate both the "sameness" and "differences" between various samples successfully $[11,14]$. Thus, we should globally consider multiple constituents in the HM extracts, and not individually consider only one and/or two marker components for evaluating the quality of the HM products.

However, in any HM and its extract, there are hundreds of unknown components and many of them are in low amount. Moreover, there usually exists variability within the same herbal materials $[4,15]$. Consequently, to obtain reliable chromatographic fingerprints that represent pharmacologically active and chemically characteristic components is not an easy or trivial work. Fortunately, chromatography offers 
very powerful separation ability, such that the complex chemical components in HM extracts can be separated into many relatively simple sub-fractions. Furthermore, the recent approaches of applying hyphenated chromatography and spectrometry such as high-performance liquid chromatography-diode array detection (HPLC-DAD), gas chromatography-mass spectroscopy (GC-MS), capillary electrophoresis-diode array detection (CE-DAD), HPLC-MS and HPLC-NMR, could provide the additional spectral information, which will be very helpful for the qualitative analysis and even for the on-line structural elucidation. With the help of the spectral information the hyphenated instruments show greatly improved performances in terms of the elimination of instrumental interferences, retention time shift correction, selectivity, chromatographic separation abilities, measurement precision [16-18]. If hyphenated chromatography is further combined with chemometric approaches, clear pictures might be developed for chromatographic fingerprints obtained. These excellent properties are the so-called dimension advantages proposed by Booksh and Kowalski [19]. A chemical fingerprint obtained by hyphenated chromatography, out of question, will become the primary tool for quality control of herbal medicines [13-15,20-37].

\section{Chromatography and chemical fingerprints of herbal medicines}

In general, the methods for quality control of herbal medicines involve sensory inspection (macroscopic and microscopic examinations) and analytical inspection using instrumental techniques such as thin layer chromatography, HPLC, GC-MS, LC-MS, near infrared (NIR), and spectrophotometer, etc. [38]. On the other hand, the methods of extraction and sample preparation are also of great importance in preparing good fingerprints of herbal medicines. In this review article, however, we shall only focus our attention on how to construct reasonably the chromatographic fingerprints and their reasonable and efficient evaluation for the purpose of quality control. As a single herbal medicine may contain a great many natural constituents, and a combination of several herbs might give rise to interactions with hundreds of natural constituents during the preparation of extracts, the fingerprints produced by the chromatographic instruments, which may present a relatively good integral representation of various chemical components of herbal medicines, are mainly concerned in this review.

\subsection{Thin layer chromatography}

TLC was the common method of choice for herbal analysis before instrumental chromatography methods like GC and HPLC were established. Even nowadays, TLC is still frequently used for the analysis of herbal medicines since various pharmacopoeias such as American Herbal Pharma- copoeia (AHP) (Upton, Santa Cruz, US, 2002), Chinese drug monographs and analysis (Wagner, Kötzting/Bayer, Wald, Germany, 1997), Pharmacopoeia of the People's Republic of China (Chemical Industry Press, Beijing, 1997), etc. still use TLC to provide first characteristic fingerprints of herbs. Rather, TLC is used as an easier method of initial screening with a semiquantitative evaluation together with other chromatographic techniques. As there is relatively less change in the simple TLC separation of herbal medicines than with instrumental chromatography, only a brief summary is given here, and for further details about TLC the readers could consult references [39-40].

TLC has the advantages of many-fold possibilities of detection in analyzing herbal medicines. In addition, TLC is rather simple and can be employed for multiple sample analysis. For each plate, more than 30 spots of samples can be studied simultaneously in one time. Thus, the use of TLC to analyze the herbal medicines is still popular [41-50,221-224]. With the help of the CAMAG video store system (CAMAG, Switerland) and TLCQA-UV methods[51], it is possible to get useful qualitative and quantitative information from the developed TLC plate. One can observe that, in reference [51], the four samples of Cordyceps sinensis from the joint products of China and Japan cooperation have more valuable medical effect compared to others as they contained the most effective component cordycepin. Moreover, with the help of image analysis and digitized technique developed in computer science, the evaluation of similarity between different samples is also possible.

In summary, the advantages of using TLC to construct the fingerprints of herbal medicines are its simplicity, versatility, high velocity, specific sensitivity and simple sample preparation. Thus, TLC is a convenient method of determining the quality and possible adulteration of herbal products.

It is worth noting that the technique of TLC are also being updated in progress. A recent paper [52] gave a very good review on this respect. It summarized the progress in forced-flow planar chromatography (FFPC) and demonstrated the importance of the different techniques like rotation planar chromatography (RPC), overpressured-layer chromatography (OPLC), and electroplanar chromatography (EPC). A simple, but powerful preparative forced-flow technique was also reported; in this technique hydrostatic pressure is used to increase mobile-phase velocity. Parallel- and serially-coupled layers open up new vistas for the analysis of a large number of samples (up to 216) for high throughput screening and for the analysis of very complex matrices. Some applications, relating to different classes of substances, were given to demonstrate the versatility of the various FFPC techniques [52].

\subsection{Gas chromatography and volatile components in herbal medicines}

It is well-known that many pharmacologically active components in herbal medicines are volatile chemical com- 
pounds. Thus, the analysis of volatile compounds by gas chromatography is very important in the analysis of herbal medicines. The GC analysis of the volatile oils has a number of advantages. Firstly, the GC of the volatile oil gives a reasonable "fingerprint" which can be used to identify the plant. The composition and relative concentration of the organic compounds in the volatile oil are characteristic of the particular plant and the presence of impurities in the volatile oil can be readily detected. Secondly, the extraction of the volatile oil is relatively straightforward and can be standardized and the components can be readily identified using GC-MS analysis. The relative quantities of the components can be used to monitor or assess certain characteristics of the herbal medicines. Changes in composition of the volatile oil may also be used as indicators of oxidation, enzymatic changes or microbial fermentation.

The advantages of GC clearly lie in its high sensitivity of detection for almost all the volatile chemical compounds. This is especially true for the usual FID detection and GC-MS. Furthermore, the high selectivity of capillary columns enables separation of many volatile compounds simultaneously within comparatively short times. Thus, over the past decades, GC is a popular and useful analytical tool in the research field of herbal medicines [53-62]. Especially, with the use of hyphenated GC-MS instrument, reliable information on the identity of the compounds is available as well (see next section for more detail). However, the most serious disadvantage of GC is that it is not convenient for its analysis of the samples of polar and non-volatile compounds. For this, it is necessary to use tedious sample work-up which may include derivatization. Therefore, the liquid chromatography becomes an another necessary tool for us to apply the comprehensive analysis of the herbal medicines.

\subsection{High-performance liquid chromatography}

HPLC is a popular method for the analysis of herbal medicines because it is easy to learn and use and is not limited by the volatility or stability of the sample compound. In general, HPLC can be used to analyze almost all the compounds in the herbal medicines. Thus, over the past decades, HPLC has received the most extensive application in the analysis of herbal medicines [63-76]. Reversed-phase (RP) columns may be the most popular columns used in the analytical separation of herbal medicines.

It is necessary to notice that the optimal separation condition for the HPLC involves many factors, such as the different compositions of the mobile phases, their $\mathrm{pH}$ adjustment, pump pressures, etc. Thus, a good experimental design for the optimal separation seems in general necessary [73,76]. In order to obtain better separation, some new techniques have been recently developed in research field of liquid chromatography. These are micellar electrokinetic capillary chromatography (MECC) [77], high-speed counter-current chromatography (HSCCC), low-pressure size-exclusion chromatography (SEC) [78], reversed-phase ion-pairing
HPLC (RP-IPC-HPLC) [79,80], and strong anion-exchange HPLC (SAX-HPLC) [81]. They will provide new opportunities for good separation for some specific extracts of some herbal medicines. On the other hand, the advantages of HPLC lie in its versatility for the analysis of the chemical compounds in herbal medicines, However, the commonly used detector in HPLC, say single wavelength UV detector, seems to be unable to fulfill the task, since lots of chemical compounds in herbal medicines are non-chromophoric compounds. Consequently, a marked increase in the use of HPLC analysis coupled with evaporative light scattering detection (ELSD) in a recent decade demonstrated that ELSD is an excellent detection method for the analysis of non-chromophoric compounds [82-84]. This new detector provides a possibility for the direct HPLC analysis of many pharmacologically active components in herbal medicines, since the response of ELSD depends only on the size, shape, and number of eluate particles rather than the analysis structure and/or chromophore of analytes as UV detector does. Especially, this technique is quite suitable for the construction of the fingerprints of the herbal medicines. Moreover, the qualitative analysis or structure elucidation of the chemical components in HM by simple HPLC is not possible, as they rely on the application of techniques using hyphenated HPLC, such as HPLC-MS, HPLC-NMR, for the analysis of herbal medicines. This topic will be further discussed later on.

\subsection{Electrophoretic methods}

Capillary electrophoresis was introduced in early 1980s as a powerful analytical and separation technique [85] and has since been developed almost explosively. It allows an efficient way to document the purity/complexity of a sample and can handle virtually every kind of charged sample components ranging from simple inorganic ions to DNA. Thus, there was an obvious increase of electrophoretic methods, especially capillary electrophoresis, used in the analysis of herbal medicines [86-108] in last decades. The more or less explosive development of capillary electrophoresis since its introduction has to a great extent paralleled that of liquid chromatography. Most of the used techniques are capillary zone electrophoresis (CZE), capillary gel electrophoresis (CGE) and capillary isoelectric focusing (cIEF). CE is promising for the separation and analysis of active ingredients in herbal medicines, since it needs only small amounts of standards and can analyze samples rapidly with a very good separation ability. Also, it is a good tool for producing the chemical fingerprints of the herbal medicines, since it has similar technical characteristics of liquid chromatography. Recently, several studies dealing with herbal medicines, have been reported and two kinds of medicinal compounds, i.e. alkaloids [95-102] and flavonoids [103-108], have been studied extensively.

In general, $\mathrm{CE}$ is a versatile and powerful separation tool with a high separation efficiency and selectivity when analyzing mixtures of low-molecular-mass components. 
However, as Shibabi and Hinsdale [109] pointed out that the fast development in capillary electrophoresis has so far been focused on the improvement of resolution and throughput rather than reproducibility and absolute precision. One successful approach to improve the reproducibility of both mobility and integral data has been based on internal standards [110]. Many papers were published on the subject over the last years but unfortunately they often only give a limited image on the real possibilities of CE in the field of fingerprinting herbal medicines. On the stony road to consistent results, not only the complexity, heterogeneity and polydispersity of herbal medicines may limit straightforward conclusions, but also many artifacts can result from the chosen separation buffer chemistry with hidden instrumental constraints. However, we believe that $\mathrm{CE}$ and capillary electrochromatography approaches would contribute to a better understanding of the solution behavior of herbal medicines, especially when additionally combined (offline or online) with the powerful spectrometric detectors.

\section{Hyphenation procedures}

In the past two decades, combining a chromatographic separation system on-line with a spectroscopic detector in order to obtain structural information on the analytes present in a sample has become the most important approach for the identification and/or confirmation of the identity of target and unknown chemical compounds. For most (trace-level) analytical problems in the research field of herbal medicines, the combination of column liquid chromatography or capillary gas chromatography with a UV-vis or a mass spectrometer (HPLC-DAD, CE-DAD, GC-MS and LC-MS, respectively) becomes the preferred approach for the analysis of herbal medicines.

It is also true that additional and/or complementary information is, in quite a number of cases, urgently required. This can be provided by, for example, atomic emission, Fourier-transform infrared (FTIR), fluorescence emission (FE), or nuclear magnetic resonance (NMR) spectrometry. It is demonstrated that, from a practical point of view, rewarding results can be obtained, since we need much more information to deal with the most complex analytical systems such as those samples from herbal medicines.

Furthermore, the data obtained from such hyphenated instruments are the so-called two-way data, say one way for chromatogram and the other way for spectrum, which could provide much more information than the classic one-way chromatography. With the help of chemometrics, a rather new discipline developed both in chemistry and statistics in the later part of the 1970s, we will definitely get more chance to deal with the difficult problems in the analysis of herbal medicines and also the problems in quality control of herbal medicines, which will be discussed in some detail in this review.

\section{1. $G C-M S$ and herbal medicines}

Mass spectrometry is the most sensitive and selective method for molecular analysis and can yield information on the molecular weight as well as the structure of the molecule. Combining chromatography with mass spectrometry provides the advantage of both chromatography as a separation method and mass spectrometry as an identification method. In mass spectrometry, there is a range of methods to ionize compounds and then separate the ions. Common methods of ionization used in conjunction with gas chromatography are electron impact (EI) and electron capture ionization (ECI). EI is primarily configured to select positive ions, whereas ECI is usually configured for negative ions (ECNI). EI is particularly useful for routine analysis and provides reproducible mass spectra with structural information which allows library searching. GC-MS was the first successful online combination of chromatography with mass spectrometry, and is widely used in the analysis of essential oil in herbal medicines [111-125].

With the GC-MS, people could produce not only a chromatographic fingerprint of the essential oil of the herbal medicine but also the information related to its most qualitative and relative quantitative composition [116-120,126,127]. Used in the analysis of the herbal medicines, there are at least two significant advantages for GC-MS, that is: (1) with the capillary column, GC-MS has in general very good separation ability, which can produce a chemical fingerprint of high quality; (2) with the coupled mass spectroscopy and the corresponding mass spectral database, the qualitative and relatively quantitative composition information of the herb investigated could be provided by GC-MS, which will be extremely useful for the further research for elucidating the relationship between chemical constituents in herbal medicine and its pharmacology in further research. Thus, in our opinion, GC-MS should be the most preferable tool for the analysis of the volatile chemical compounds in herbal medicines.

\subsection{HPLC-DAD, HPLC-MS and others}

HPLC-DAD has become a common technique in most analytical laboratories in the world now. With the additional UV spectral information, the qualitative analysis of complex samples in herbal medicines turns out to be much easier than before. For instance, checking peak purity and comparing with the available standard spectrum of the known compound to the one in the investigated sample. Especially, with the introduction of electrospray mass spectrometry, the coupling of liquid chromatography and mass spectrometry has opened the new way to widely and routinely applied to the analysis of herbal medicines. HPLC chromatographic fingerprints can be then applied for documentation of complete herbal extracts with more information and on-line qualitative analysis becomes possible. Several valuable review articles dealing with LC-MS and its application in the analysis of botanical 
extracts have been published [128-135], In last decades, the increasing usage of LC-MS and HPLC-DAD in the analysis of herbal medicines is quite obvious [136-146]. Several good reviews have been published for the analysis of the bioactive chemical compounds in plants and herbal medicines, in which the technique used most is HPLC, especially the hyphenated HPLC techniques [147,148]

Moreover, combined HPLC-DAD-MS techniques take advantage of chromatography as a separation method and both DAD and MS as an identification method. DAD and MS can provide on-line UV and MS information for each individual peak in a chromatogram. With the help of this hyphenation, in most cases, one could identify the chromatographic peaks directly on-line by comparison with literature data or with standard compounds, which made the LC-DAD-MS becomes a powerful approach for the rapid identification of phytochemical constituents in botanical extracts, and it can be used to avoid the time-consuming isolation of all compounds to be identified [149]. Recently, the hyphenation between HPLC and NMR are also available [150-154], which might become a vital and an attractive analytical tool for the analysis of drugs in biological fluids and for the analysis of herbal medicines [155]. In fact the tendency of the hyphenation or multi-hyphenation of the chromatography with the common used four spectroscopic detectors, say UV, Fouriertransformation infrared spectrum, MS and NMR, for structure elucidation of chemical compounds, is in progress. A recent paper [156] gave a good review on this. A "total analysis device" has been recently demonstrated in the case of on-line HPLC-UV(DAD)-FT-IR-NMR-MS analyses.

It may be worth noting that the electrode array hyphenated with HPLC is also in progress [157]. As electrochemical detection was described to be superior to UV-vis and fluorescence spectroscopy for determination of some chemical compound, like polyphenols in trace levels [158-160], very sensitive determination was achieved by using a multi channel electrochemical detector (CoulArray). A two-way chromatogram is obtained, since the detector has 12 (or more) electrodes in series set incrementally to different potentials. Thus, similar to the UV-vis spectra, the hydrodynamic voltammogram can be used for peak purity checking and peak identification.

\subsection{Hyphenation of $C E$}

The situation of the $\mathrm{CE}$ analysis in hyphenation development is somewhat like HPLC analysis. The hyphenated CE instruments, such as CE-DAD, CE-MS and CE-NMR, all appeared in the past decades. The techniques have also quickly been used for the analysis of the samples from herbal medicines [161-167]. On-line coupling of capillary electrophoresis to mass spectrometry and other spectrometry allows both the efficient separation of CE and the specific and sensitive detection to be achieved. Furthermore, the artifacts happened in CE measurements might be overcome with the help of some information handling technique, such as some methods developed in chemometrics, since one could use with the additional information from spectra to correct the artifacts from the chosen separation buffer chemistry or from hidden instrumental constraints (see later for more details).

In sum, as the hyphenated techniques in chromatographic and electrophoretic instruments develop, our ability of analysis of herbal medicines, both in qualitative and quantitative respects, and our ability of quality control of herbal medicines will become stronger and stronger. We are quite sure that we will have a very prospective future for quality control of herbal medicines.

\section{Information features of chromatographic fingerprints of herbal medicines}

From the discussion above, we can see clear that there are many chromatographic techniques, including the hyphenated chromatographies, available for us to do the instrumental analysis of herbal medicines, and to construct further their fingerprinting. The problem here is that how could we efficiently and reasonably evaluate such-obtained analytical results and/or the fingerprints of the herbal medicines and how could we use the information obtained from chromatographic analysis to address further the problem of quality control for herbal medicines, which are just the topic we will discuss in the following sections.

\subsection{Phytoequivalence and chromatographic fingerprints of herbal medicines}

In general, one could use the chromatographic techniques to obtain a relatively complete picture of an herbal, which is in common called chromatographic fingerprints of herbal medicines to represent the so-called phytoequivalence. The following results shows some examples.

Fig. 1 shows the total ionic chromatograms (TIC) of essential oils in Cortex cinnamomi from four producing areas, say: (a) Zhaoqing, Guangdong province, China; (b) Yulin, Guangxi province, China; (c) Yunnan province, China; and (d) Vietnam, by GC-MS [17]. There are, of course, some differences in the profiles. However, seen from these profiles, phytoequivalence is obvious for $C$. cinnamomi. The other example is shown in Fig. 2, in which there are chromatograms of methanol extracts of Erigeron breviscapus from 32 different producing places in the same province by HPLC detected at wavelength $280 \mathrm{~nm}$. The integrated feature of the chromatographic fingerprints of herbal medicines can be clearly seen from these examples.

Obtaining a good chromatographic fingerprint representing the phytoequivalence of a herb depends several factors, such as the extracting methods, measurement instruments, measurement conditions, etc. In fact, if we want to obtain an informative fingerprint of a herbal medicine, we need to have a good extracting method, with which we could fortunately obtain almost all the pharmaceutically active compounds to 

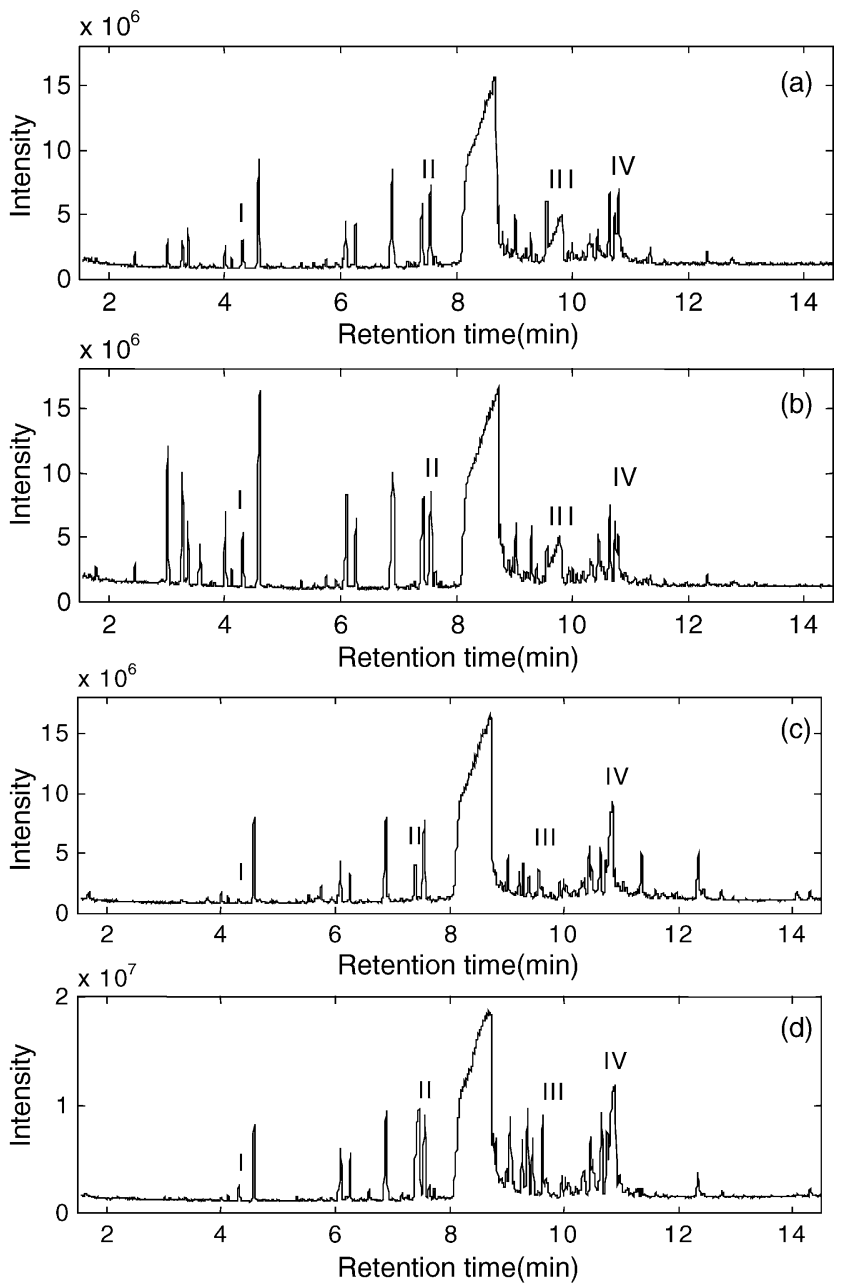

Fig. 1. Total ionic chromatograms of essential oils in Cortex cinnamomi from four different producing areas: (a) Zhaoqing, Guangdong province, China; (b) Yulin, Guangxi province, China; (c) Yunnan province, China; and (d) Vietnam.

represent the integrity of the herbal medicine. Furthermore, a chromatogram with good separation and a representative concentration profile of the bioactive components detected by a proper detector are also required. Thus, how to obtain a high quality chromatographic fingerprint of as more as possible information of the herbal medicines is an important task for chemists and pharmacologists. Some researches have been done along with this direction [168-172]. In order to understand bioactivities and possible side effects of active compounds of the herbal medicines and to enhance product quality control, it seems that one needs to determine most of the phytochemical constituents of herbal products so as to ensure the reliability and repeatability of pharmacological and clinical research. Suppose that we have obtained some fingerprints, how to evaluate the information contents of the chromatographic fingerprints of herbal medicines reasonably and efficiently is the second step for the quality control purpose.

\subsection{Information contents of fingerprints of herbal medicines}

It is obvious that a chromatographic fingerprint of a herbal medicine is a multivariate system, since in general it embraces most of the phytochemical constituents of herbal products. From a point of view of multivariate, the information content of a chromatogram with lots of peaks might be calculated by means of various approaches [168-172]. However, as for these methods, the signal intensity, retention time, peak area and/or peak height of each independent peak without overlapping should be all taken into consideration, the calculation burden is also rather heavy. Moreover, if a chromatographic peak is overlapped with its adjacent peak(s), the calculation of the information content will become complex. Vertical splitting is conventionally used for this situation and both peaks on two sides of an overlapping peak cluster are taken as pure ones. Out of question, this approximate treatment on a chromatogram with some overlapping peak clusters will cause some errors on calculation of information content.

In fact, a chromatographic fingerprint, which is a concentration distribution curve of several chromatographic peaks, could be regarded as a continuous signal determined by its chromatographic shape. According to information theory [173], the information content of a continuous signal might be simply expressed as the following formula:

$\Phi=-\sum p_{i} \log p_{i}$

where $p_{i}$ is the positive real numbers of probability property, say $\sum p_{i}=1$. Based on this idea, we proposed recently a simple method [173] to calculate the information content $(\Phi)$ for chromatographic fingerprints of herbal medicines as shown in the following equation, that is

$\Phi=-\sum\left(\frac{x_{i}}{\sum x_{i}} \log \left(\frac{x_{i}}{\sum x_{i}}\right)\right)$

where $x_{i}$ is the real chromatographic response of the chemical components involved in the chromatographic fingerprint under study. Here, the normalization of $x_{i}$ divided by their sum is to make the chromatogram investigated be of probability property. In theory, if and only if $x_{i}$ with unchangeable variance is characterized by normal distribution can its information content $\Phi$ reach its maximum [174]. Under an ideal situation, all the chromatographic peaks from a chromatogram can be separated completely and each peak confined to a narrow zone might correspond to a normal distribution profile [175]. A chromatographic fingerprint with all of peaks just completely separated should be featured by maximal information content. Further separation cannot provide any more information and becomes unnecessary. On the contrary, if any of chromatographic peaks is overlapped with its adjacent one(s), this peak will surely show non-Gaussian normal distribution and therefore undoubtedly cause a loss of the information content. There might be, at least, two advantages of the calculation of the information content 


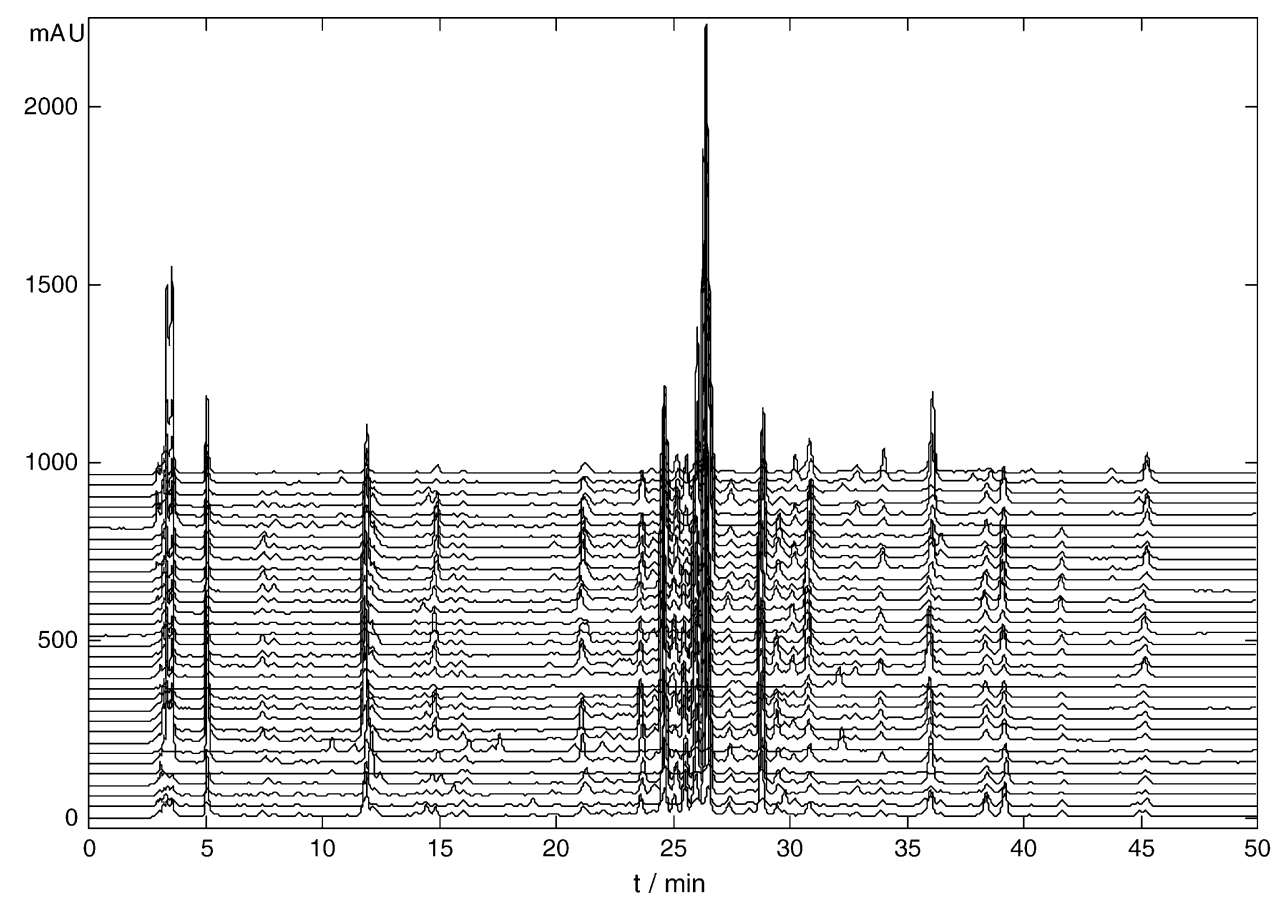

Fig. 2. Original HPLC chromatograms of 33 herbal samples of Erigeron breviscapus at wavelength $\lambda=280 \mathrm{~nm}$.

of complex chromatograms over the available approaches [168-172]. First, the method uses the whole chromatogram with a simple normalization, thus it is not necessary to identify the retention time, peak intensity, peak width, peak area and/or peak height for all the peaks identified. The calculation burden is reduced significantly. Moreover, the theoretic background of the method is simple and reasonable. For more details, the readers can consult the reference [173].

\subsection{Correction of retention time shift of fingerprints of herbal medicines}

When one deals with several chromatographic fingerprints obtained from the same herbal medicine or from different sources, the first step of our task might be to evaluate their similarity and difference between them. However, correction of retention time shift of the fingerprint of herbal medicines should be taken into consideration first, since some types of variation sources are inevitably encountered from one chromatogram to another [7-13,166-182]. Under this situation, some unacceptable results, one of which is imposed by the retention time shifts, will be produced. Unfortunately, how the retention time shifts are caused by the variation sources is very complex. It might be due to (1) the degradation of the stationary phase, especially, the low stability of silica and silica-based supports at high $\mathrm{pH}$ values and the collapse of C18 bonded phase because of a highly polar mobile phase; (2) minor changes in mobile phase composition caused by temperature and pressure fluctuations, variations in flow-rate and gradient dispersion; (3) some problems involved in the detectors, for example, a wavelength shift in the UV spectrometer, a spectroscopic intensity variation and the misalignment of the monochromator; (4) the column overloading on account of the great injected amount or some components with high concentration; (5) the possible interaction between analytes; (6) other unknown shifts in the instrument. If these disadvantageous cases are in existence during the chromatographic runs, the retention times will be subsequently shifted.

When such retention time shifts occur in chromatographic fingerprints, it is great difficult to conduct data processing, for example, the construction of common chromatographic models of all samples investigated, the similarity comparison between chromatographic fingerprints and pattern recognition based on principal component analysis (PCA) since multivariate analysis with entire chromatographic profiles as input data is very sensitive to even minute variations [49]. As a result, in order to make up a consistent data, it is necessary to detect the retention time shifts of chromatographic fingerprints and then the chromatographic profiles should be adjusted along the retention time direction by means of synchronizing the retention times of the chromatographic peaks from the same components.

During the past decades, several kinds of useful approaches have been developed for peak synchronization in chromatographic profiles [176-182]. Some of them corrected the retention time shifts by making internal standards added or marker peaks coincide in all chromatograms under study [177-179]. Among them, the technique in ref. [179] was recently developed. At its first step, chromatographic peaks were identified by setting a retention time window in both of the sample and target chromatograms, and then a list of their retention times was generated. Here, the window meant the maximum shift to be considered. Clearly, the choice of the window size was critical to this technique. However, if the re- 
tention time shifts are very serious for complex systems like herbal medicines, the selection of the optimal peak-matching windows might not be a trivial task. In refs. [176,180-182], some objective functions on the correlation between the target and sample chromatograms were optimized and then the sample chromatographic profiles were aligned with the target. In general, the methods must be very efficient and elegant if the samples investigated are quite similar in the concentration profiles of the chemical components. However, if the concentration profiles change greatly for the complex samples such as herbal medicines from the different producing places and/or from the various harvest seasons, wrong results might be obtained by simply seeking the optimal correlation coefficient between the chromatograms. It is because that the correlation coefficient is influenced greatly by the big peaks in the chromatographic profiles. In this case, the maximal correlation coefficient does not certainly represent the best correction for the retention time shifts [183]. However, if the data from the hyphenated chromatographic instruments, such as HPLC-DAD, GC-MS, etc. the correction of retention time shift of the fingerprint of herbal medicines will become much clear and easy, which we will discuss later on.

\section{Evaluation of chemical fingerprints of herbal medicines}

In the early chemometric research, chromatographic data were commonly first transformed to retention time-peak area data matrices including only selected peaks, whether the identity of the peaks are known or not. The data such obtained were then used to do the processing, that is, the calculation of similarity or dissimilarity between the fingerprints and the analysis of principal component analysis [184-186].

However, as pointed by Nielsen [176], "The quality of the data (including only retention time-peak area data) relies on peak detection (integration) and on how the peaks are selected for the data analysis. It can be very difficult to select an optimal set integration parameters for chromatograms obtained from analysis of complex samples which easily contain more than 100 peaks. Furthermore, the selection and extraction of peaks to include in data analysis is difficult, partly subjective and a large amount of the data in the chromatograms are discarded. The disadvantages of peak detection and integration, and of the introduction of a subjective peak selection can be avoided by using all collected data points in the chemometric analysis." Thus, following the suggestion of Nielsen, the entire chromatographic profiles were utilized to perform direct chemometric analysis. The analysis can be easily done with the help of proper techniques of data compression, such as the technique of wavelet or Fourier transformation [187-189], if necessary. Furthermore, another advantage of taking the entire chromatographic profile to perform direct chemometric analysis is that the peak shape can be included in data analysis, which will make the pretreatment of overlapping peaks much easier when one does evaluation of the fingerprints. Of course, the chromatographic profiles should properly aligned to compensate for minor drifts in retention times before one does the fingerprint evaluation and chemoemtric analysis for the purpose of quality control.

\subsection{Fingerprints and quality control in herbal medicines}

As stated above, one or two markers or pharmacologically active components were currently employed for evaluating the quality and authenticity of a herbal medicine. This kind of the determination, however, does not give a complete picture of an herbal product and therefore it will definitely fail to do the identification of false and true plant extraction. In the following an example will be given to illustrate the situation.

Fig. 3 shows 18 fingerprints of Ginkgo biloba extractions, which were purchased from several pharmaceutical stores, vendors/companies and collected from various producing areas in the mainland of PR China. All of these samples were supposed to meet the standard measured by UV spectroscopy at wavelength of $318 \mathrm{~nm}$ with satisfactory absorbance (old standard method for quality control of G. biloba extraction in China), among which standard extract EGb761 was friendly donated to one of authors from Guangzhou Institute for Drug Control, PR China by a Frenchman from Beaufour-Ipsen Company in France with a satisfactory fingerprint pattern. Analytical grade methanol and phosphoric acid use for mobile phase were purchased from chemical reagents factory of Guangzhou, Guangdong, PR China. The other involved reagents were also of analytical grade. Ultrapure water $(18.2 \mathrm{M} \Omega$ ) was obtained by means of a Milli-Q apparatus by Millipore Corporation (France) and was used for mobile phase preparation. The mobile phase was vacuum filtered through a filter of $0.45 \mu \mathrm{m}$ pore size.

From the plot, it is difficult to find some false one. But if we simply do the PCA upon the fingerprints, the results are shown in Fig. 4. It can be easily seen from the plot that samples marked by numbers $1-3$ are clearly outliers. Thus, if we pick up the fingerprints of samples 2 and 3 (see Fig. 5C and D) and comparing them with the fingerprints (see Fig. 5A and B) of the standard extract EGb761 (number 17 in Fig. 4) and the other sample (number 8 in Fig. 4), we can easily find the difference between them. The peak in the fingerprints of samples 2 and 3 around the retention time of $10 \mathrm{~min}$ is much higher than the one in the standard extract EGb761 and sample 8. This peak is rutin. In fact, rutin was added in the three outlier samples, say samples $1-3$, in order to meet the old standard of enough absorbance. They are quite different from the real G. biloba extractions as shown in Fig. 5A. From this example, we can see that the technique of fingerprint could really identify the false herbal products.

\subsection{Similarity of fingerprints}

The construction of chromatographic fingerprints aims at evaluating the quality of HMs. As discussed above, the fundamental reason of quality control of herbal medicines is based 


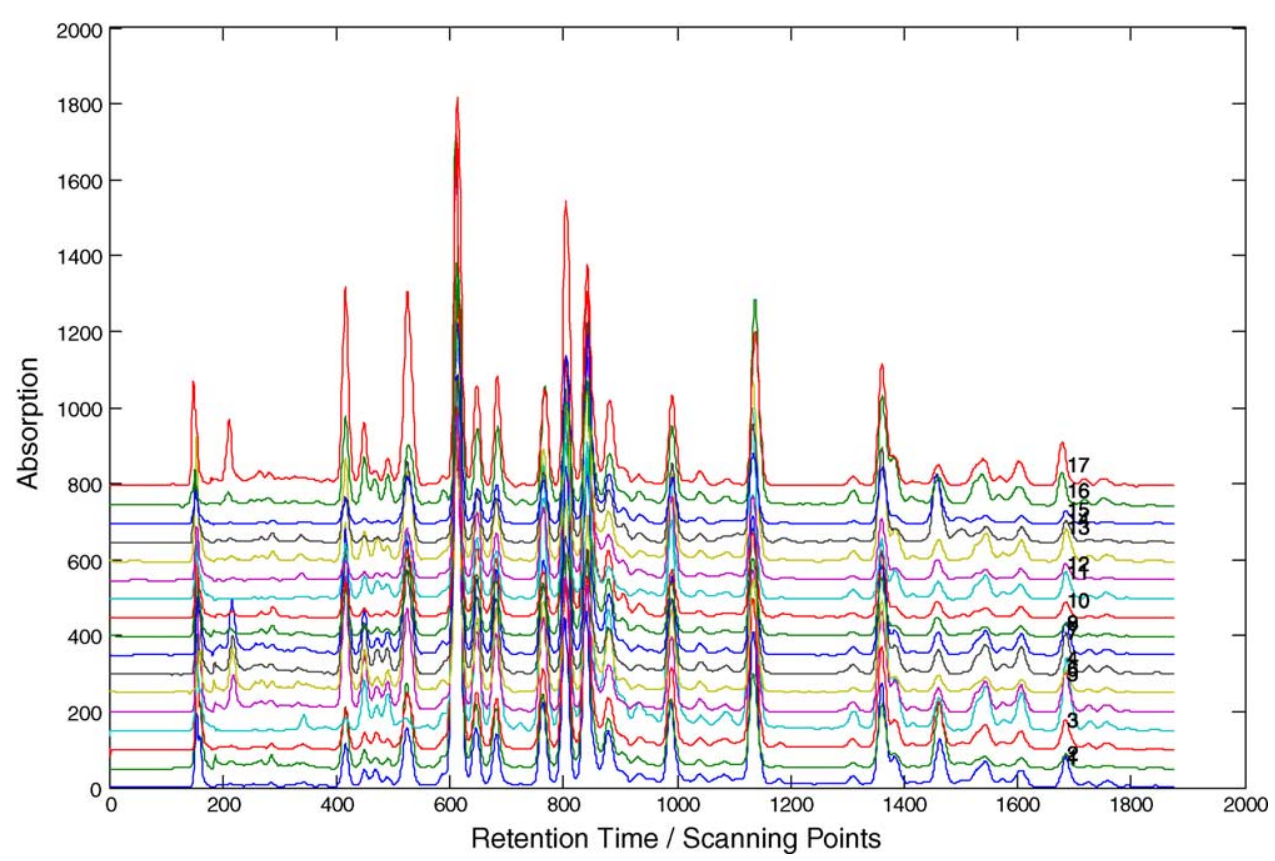

Fig. 3. Ginkgo biloba extractions were purchased from several pharmaceutical stores, vendors/companies and collected from various producing areas in the mainland, PR China. Standard extract EGb761 (No.18) was friendly donated to one of authors from Guangzhou Institute for Drug Control, PR China by a Frenchman from Beaufour-Ipsen Company in France.

on the concept of phytoequivalence of herbs, and then to use this conception to identify the real herbal medicine and the false one, and further to do quality control. Thus, the intuitive evaluation method is to compare the similarities and/or differences of the chromatographic fingerprints' shape. As a result, both the separation degrees and concentration distribution of components involved in a chromatographic fingerprint is also taken into consideration for this evaluation. The most commonly used standards for evaluation of similarity of the multivariate systems are correlation coefficient and congru-

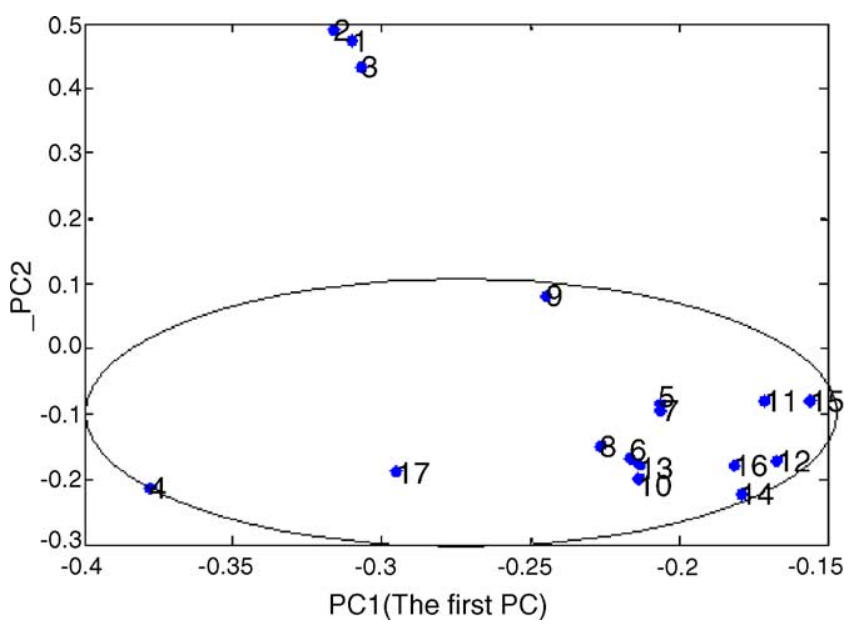

Fig. 4. The score plot obtained by principal components analysis where PC1 means the scores coordinates of principal component 1 and PC2 the ones of principal component 2 . ence coefficient as expressed by the following two formulae correlation coefficient :

$$
r_{1}=\frac{\sum\left(x_{i}-\bar{x}\right)\left(y_{i}-\bar{y}\right)}{\left(\sum\left(x_{i}-\bar{x}\right)^{2}\right)^{2}\left(\sum\left(y_{i}-\bar{y}\right)^{2}\right)^{2}} \quad(i=1,2, \ldots, n)
$$

congruence coefficient :

$$
r_{2} \frac{\sum x_{i} y_{i}}{\left(\sum\left(x_{i}\right)^{2}\right)^{1 / 2}\left(\sum\left(y_{i}\right)^{2}\right)^{1 / 2}} \quad(i=1,2, \ldots, n)
$$

where $x_{i}, y_{i}$ are the $i$ th elements in two different fingerprints, say $x$ and $y$, respectively and $n$ is the number of the elements in the fingerprints. $\bar{x}$ and $\bar{y}$ are the mean values of the $n$ elements in fingerprints $x$ and $y$, respectively, that is,

$$
\begin{aligned}
& \bar{x}=\frac{\sum x_{i}}{n} \\
& \bar{y}=\frac{\sum y_{i}}{n}
\end{aligned}
$$

The relationship within a set of chromatographic fingerprints could be currently analyzed through comparison in terms of similarity or dissimilarity of the objects with a certain reference, presented as correlation coefficient or congruence coefficient. In order to illustrate the situation, the example used in the above subsection is now applied to address such a problem. Table 1 list the correlation coefficients and congruence coefficients of the samples compared with the median spectrum of the whole 17 samples. From the table, we can see that the three false extractions have the smallest similarity values, 

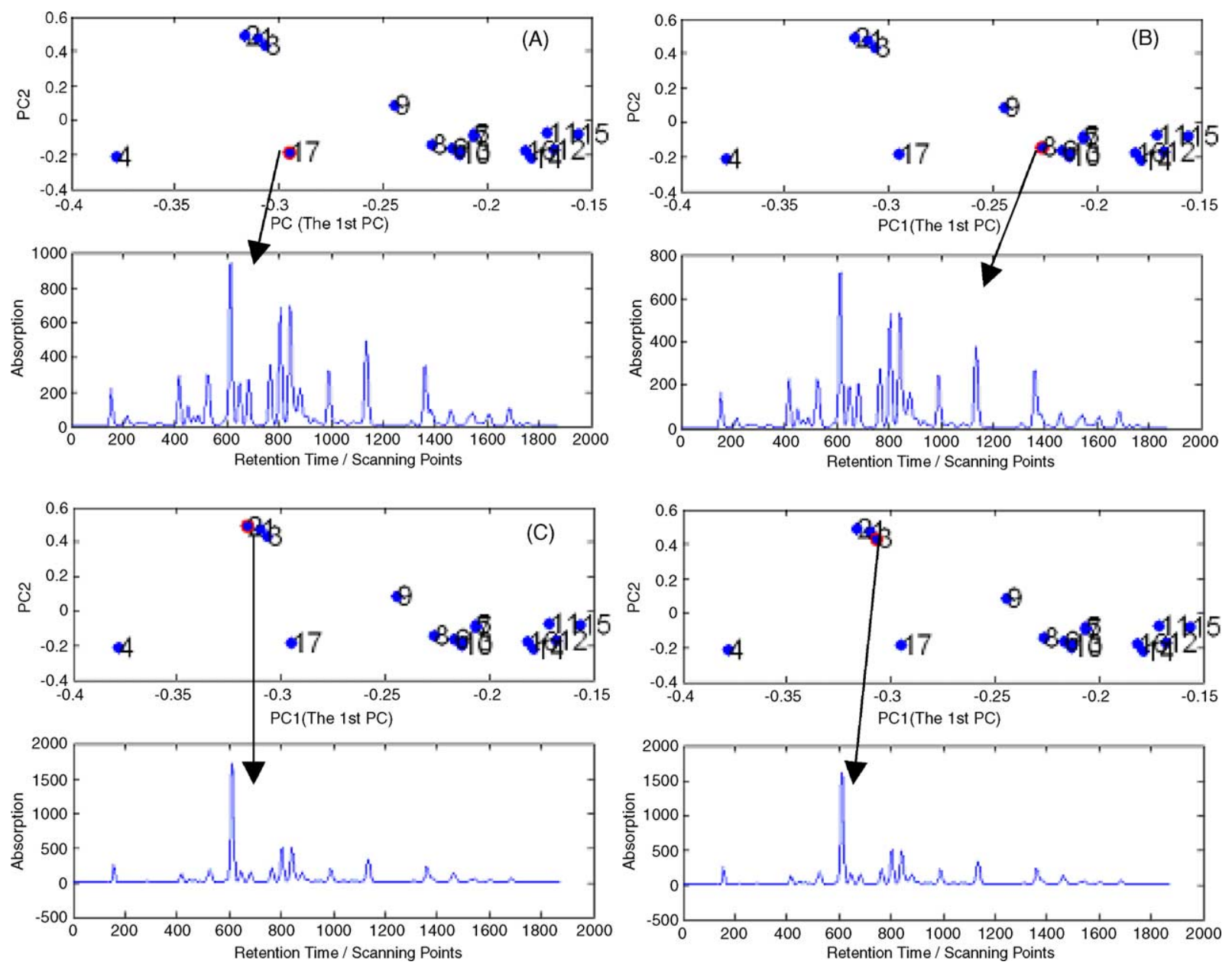

Fig. 5. The relationship between the projection points of score plot of PCA and the corresponding original fingerprints for samples $17,8,2$ and 3 , respectively.

Table 1

Correlation coefficients and congruence coefficients of the samples compared with the median spectrum of the whole 17 samples

\begin{tabular}{lll}
\hline Sample no. & Correlation coefficients & Congruence coefficients \\
\hline 1 & 0.8795 & 0.8939 \\
2 & 0.8795 & 0.8899 \\
3 & 0.8935 & 0.9051 \\
4 & 0.9349 & 0.9474 \\
5 & 0.9511 & 0.9604 \\
6 & 0.9469 & 0.9574 \\
7 & 0.9552 & 0.9637 \\
8 & 0.9779 & 0.9821 \\
9 & 0.9524 & 0.9568 \\
10 & 0.9158 & 0.9269 \\
11 & 0.9869 & 0.9895 \\
12 & 0.9506 & 0.9593 \\
13 & 0.9753 & 0.9801 \\
14 & 0.9479 & 0.9581 \\
15 & 0.9590 & 0.9640 \\
16 & 0.8979 & 0.9187 \\
17 & 0.9436 & 0.9547 \\
\hline
\end{tabular}

which seems to suggest that the similarity estimation may be used as a standard together with the original fingerprint, even this is very simple.

\subsection{Chemical pattern recognition and classification evaluation}

As discussed above, the relationship within a set of chromatographic fingerprints could be currently analyzed through comparison in terms of similarity or dissimilarity of the objects with a certain reference, presented as correlation coefficient or congruence coefficient, etc. But, it has been aware that there are two problems for this comparison: how to achieve the reasonable reference (comparing standard) and to what extent the investigated object is similar with the reference. Popularly, the reference may be derived either from standard extract of herbal medicine or proportioned mixture of herbal medicine (e.g. EGb761) or from computation by some mathematical methods (for the example above, the median chromatographic fingerprint of whole samples is taken, since there are three outliers in the samples investigated). However, it is well known that natural products derived from herbal medicines with inherent "uncertainty" feature of its 
secondary metabolic substances, to define an absolute reference fingerprint by simply calculating their mean or median for one kind of herbal medicine seems somewhat subjective. From this point of view, the conception of class of one herbal medicine seems to be more reasonable. Thus, the chemical pattern recognition methods, such as K-nearest neighbors (KNN) [190,192] and soft independent modeling of class analogy (SIMCA) [193], etc. should be taken into consideration for reasonable definition of the class of the herbal medicine [194-196]. In fact, several researchers in China had worked on the concepts of using chemical analytical and chromatographical fingerprinting to measure the consistency of raw Chinese medicinal herbs and composite formula with the application of fuzzy clustering analysis of HPLC pattern in the early 1990s [225].

On the other hand, the (dis)similarities of herbal objects with the reference often undertake themselves to a qualified threshold [191], which is not so easy to define. Although such a comparison attaches importance to the integral relationship of the fingerprints, sometimes masking and swamping effects might occur either explicitly or implicitly. The masking effect is that an unexpected sample is undecided because of high similar value (e.g. the identification of three species of Coptis chinensis, C. teet-Oides C. Y. Cheng, and C. deltoidea C. Y. Cheng et Hsiao from herb Rhizoma coptidis). The swamping effect encompasses wrongly discriminating a desirable sample illegal on account of low similarity with the reference influenced by the diversity of chromatographic compositional distribution (e.g. the determination of herb Houttuynia cordata Thunb. from different sources). To avoid these effects as much as possible, a method based on PCA after necessary data transformations [219]. The method has been demonstrated that PCA with standard normal variate transformation of data led to meaningful classification of 33 different E. breviscapus herbal samples (see Fig. 2). The result was also corroborated by variance squares discriminant method. The quality of herbal objects was further evaluated, and the causes of this fact have been explained from a chemical point of view. The other method [220] is based on secured principal component regression ( $\mathrm{SPCR}$ ) that was originally developed for detecting and correcting uncalibrated spectral features newly emerging in spectra after the PCR calibration [196,197]. It can detect and consider unexpected chromatographic features for quality evaluation of herbal samples from the point of view of analyzing fingerprint residual.

\section{Qualityfication and validation of two-way data from hyphenated chromatographies by chemometrics}

In general, the data generated by the hyphenated instruments are matrices with every row being a spectrum and every column a chromatogram at some wavelength, wavenumber or m/e unit as illustrated in Fig. 6. The data obtained by such hyphenated instruments in chemistry is generally called twoway or two-dimensional data. In common, the size of the data

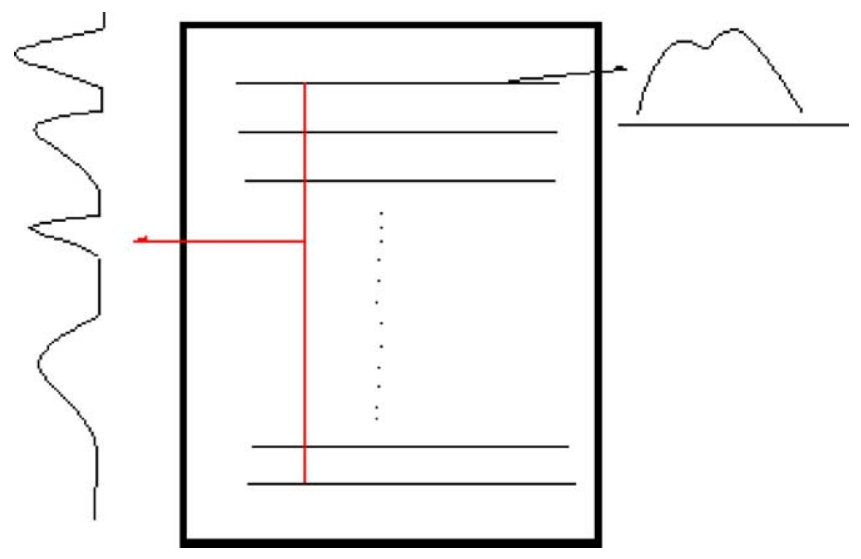

Fig. 6. Illustration of two-dimensional data from hyphenated instruments.

matrix such-obtained is rather big, sometimes it can be more than 40 megabytes. Thus, this is a really a new challenge for modern analytical chemistry to deal with the chemical information embedded in it.

However, data derived from hyphenated analytical techniques have several advantages over the classic one-way chromatograms. Firstly, the two-way data matrix contains both information from spectra and chromatograms, which will make on-line structure identification of some interested compounds possible; secondly, the two-way data has so-called dimension advantages proposed by Booksh and Kowalski [19], which will make on-line comparison of overlapping chromatographic peaks possible; finally, the hyphenated technique might enhance the chromatographic separation ability by the additional spectral information, since one could easily find some useful component selectivity with the help of chemometric local rank analysis methods, which can only be used for two-way chromatographic data but never for one-way chromatographic data. For more detail, the readers can consult reference [218].

How to use these advantages from the two-way data to address the problems in evaluation of the fingerprints of herbal medicines and the problems in quality control of herbal medicines, which is just the topic we want to discuss in the following subsections. There are, to our best knowledge, still many different kinds of difficult problems unsolved in the research field of herbal medicines. For instance, there may be no big problem for evaluating the fingerprints from the same instruments and/or with the same batch of the herbal products as shown above in this review. However, if we get some fingerprints from different laboratories and/or from the same kind of column, say C-18 for example, but from different companies. Could we still evaluate them reasonably? Moreover, could we do the on-line comparison among the fingerprints of some overlapping chromatographic peaks and/or of big diversity? For instance, if we get some fingerprints from different extraction methods and/or from different herbal medicines, is it possible for us to see whether we have got the same phytochemical constituents or not in order to understand their bioactivities and possible side effects of these herbal prod- 
ucts and consequently to enhance product quality control? With the help of the two-way data from the hyphenated chromatographies and chemometric methods [198] recently developed, the answer is positive.

\subsection{Spectral correlative chromatogram and its applications}

As stated above, it is very important for assessment of the quality of various samples to determine the presence or absence of interested components among the different chromatographic fingerprints. They may be obtained either from same herbs or from different ones under the same or similar chromatographic separated conditions. Moreover, another problem is the shift of retention time of some interested peaks of various fingerprints due to inevitable possibility in quality control, such as the fingerprints from different laboratories and the experimental columns from different vendors despite the same type stationary-phase characteristics. This maybe lead to erroneous assess of quality of medical samples. Let us see an example shown in Fig. 6.

$\boldsymbol{x}_{1}$ and $\boldsymbol{x}_{2}$ shown in Fig. 7A are the chromatograms of twodimensional data sets of $\boldsymbol{X}_{1}$ (of the G. biloba samples obtained
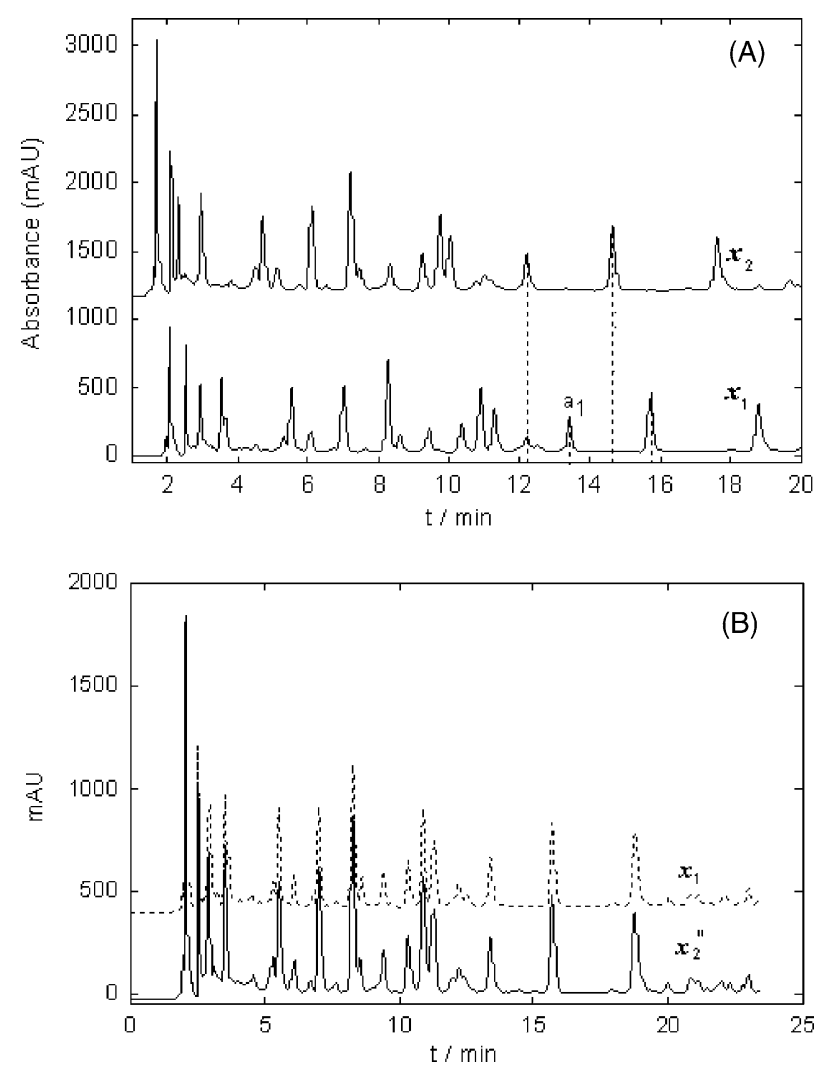

Fig. 7. Comparison of chromatograms $\boldsymbol{X}_{1}$ and $\boldsymbol{X}_{2}$ measure by the same kind of column but from different companies: $\boldsymbol{X}_{1}$ (Angilent Inc.) and $\boldsymbol{X}_{2}$ (Waters Inc.). (A) Original chromatograms of samples $\boldsymbol{X}_{1}$ and $\boldsymbol{X}_{2}$ at wavelength $260 \mathrm{~nm}$, respectively. (B) Original chromatograms of samples $X_{1}$ and chromatogram $\boldsymbol{X}_{2}^{\prime \prime}$ of sample $\boldsymbol{X}_{2}$ after correction of retention time shifting by local least-squares technique. from HPLC-DAD through the column made in Angilent Inc.) and $\boldsymbol{X}_{2}$ (through the column made in Waters Inc.) at wavelength $260 \mathrm{~nm}$, respectively. It can be intuitively seen that the retention time of eluting components of them shift rather seriously, which trouble right identification of them by directly comparing their chromatographic fingerprints.

In order to deal with such a kind of problem a technique named spectral correlative chromatogram (SCC) was developed [199]. The idea of SCC is quite simple, that is, the same chemical component should be of the same spectrum no matter what they are eluted through diverse chromatographic columns. Thus, one could use the spectral information to pick up the interested compound from the other two-way chromatograms. The whole procedure goes in the following steps: (1) assess peak purity of an interested compound and then acquire its spectrum; (2) identify correlative components in the other fingerprint by series correlation coefficients between the spectrum obtained above and the spectra at every scan point for the other two-way chromatogram; (3) get a curve (named SCC) of correlation coefficients at every scan point in the direction of retention time and further validate the result from the second step combining the information of local chromatographic cluster where targets exist; (4) eventually assess the similarity and/or difference of the chromatographic fingerprints after correcting the time shift of correlative components in a piecewise way by using local least squares. Fig. 8 shows an example of this procedure. Pure component $a_{1}$ in $\boldsymbol{X}_{1}$ indicated in Fig. 8 was taken as an example to illustrate this procedure. The obtained SCC is shown in Fig. 8B. As could be seen from Fig. 8, both the correlation coefficients $r\left(a_{1}, a_{2}\right)$ and $r\left(a_{1}, b_{2}\right)$ corresponding to components $a_{2}$ and $b_{2}$ of $\boldsymbol{X}_{2}$ were larger than others and quite close to 1 , say $r\left(a_{1}\right.$, $\left.a_{2}\right)=0.9998$ and $r\left(a_{1}, b_{2}\right)=0.9940$. Thus, components $a_{1}$ and $a_{2}$ were correlative, even though they were unknown and there were some shift of their retention time. Furthermore, spectrum-dependent principle of identification of substance decided the result. Fig. 7C and D (lower part) exhibits the spectra $\boldsymbol{s}_{a_{1}}, \boldsymbol{s}_{a_{2}}$ and $\boldsymbol{s}_{b_{2}}$. It is obvious that $\boldsymbol{s}_{a_{1}}$ and $\boldsymbol{s}_{a_{2}}$ are entirely consistent and factually identical, whereas $\boldsymbol{s}_{a_{1}}$ and $\boldsymbol{s}_{b_{2}}$ are something different. Similarly, other correlative components could also be obtained, as listed in Table 2. With the correlative information available, the retention time shift can be easily corrected by local least squares taking $x_{1}$ as a target reference [199], The results after shift correction are shown in Fig. 7B. From this plot, we can see that the shift of retention time can be corrected reasonably. Along with this direction, the spectral correlative chromatogram for multi-components' comparing could be also possible and conducted [200].

\subsection{On-line comparison by chemometric methods}

In order to understand the bioactivities and possible side effects of some herbal products and consequently to enhance product quality control one might be asked to compare directly some samples to see whether there are same phytochemical constituents in the different samples. For instance, 

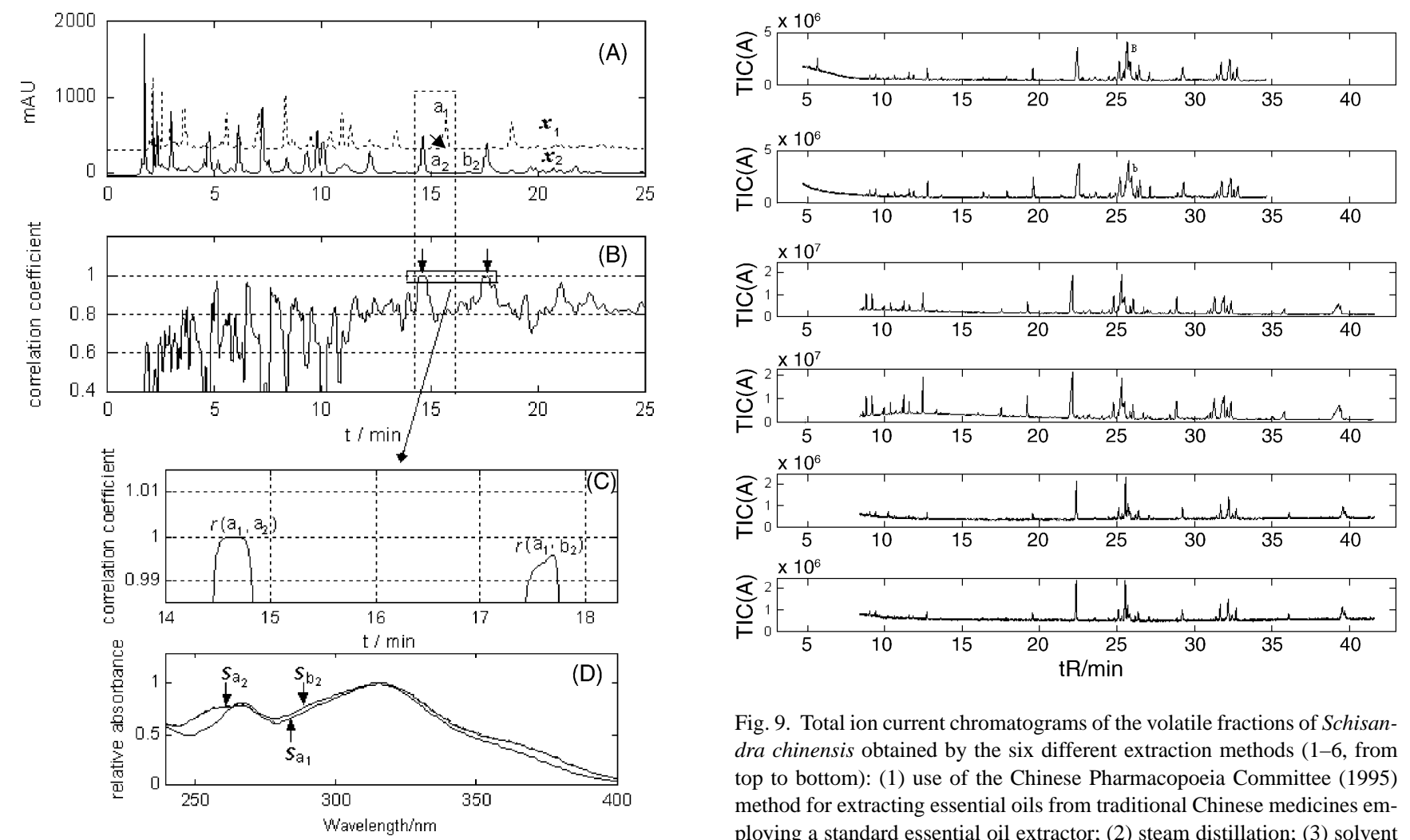

Fig. 9. Total ion current chromatograms of the volatile fractions of Schisandra chinensis obtained by the six different extraction methods (1-6, from top to bottom): (1) use of the Chinese Pharmacopoeia Committee (1995) method for extracting essential oils from traditional Chinese medicines employing a standard essential oil extractor; (2) steam distillation; (3) solvent extraction with petroleum ether using an ultrasonic extractor; (4) Soxhlet extraction with petroleum ether; (5) solvent extraction with diethyl ether using an ultrasonic extractor; and (6) Soxhlet extraction with diethyl ether.

Fig. 8. Illustration of the procedure of spectral correlative chromatogram Two original samples, say $\boldsymbol{X}_{1}$ and $\boldsymbol{X}_{2}$, involved in comparison, in which a peak indicated by $a_{1}$ is the component spectrum to be searched by SCC. (B) The spectral correlative chromatogram obtained for component $a_{1}$. (C) Enlarged part of spectral correlative chromatogram around retention time range of 14-18.5 min. (D) Spectral comparison of spectra $\boldsymbol{s}_{a}, \boldsymbol{s}_{a}$ and $\boldsymbol{s}_{a}$, respectively.

if the extraction method is changed the chemical compositions of the products will also change? Fig. 9 shows such an example, in which the total ion current chromatograms of the volatile fractions of $S$. chinensis derived from six different extraction methods are presented. It may be observed that the major section (retention time region from 15 to $35 \mathrm{~min}$ ) is quite similar in each of the six chromatograms. In order to carry out a more detailed analysis, however, it is necessary to compare qualitatively the results from the six methods. There are three difficulties in comparing such results: (1) it is difficult to conclude if the same compounds are present in overlapping chromatographic peaks; (2) it is difficult to confirm whether a peak with a given retention time represents the

same compound in different extractions or whether there is significant chromatographic drift between runs (see top part in Fig. 10 for details); and (3) the chromatographic background makes MS matching difficult. In order to overcome these problems, the mass spectral information together with chemometric methods seems to be necessary to introduced.

Subwindow factor analysis (SFA) is just such a chemometric resolution method [201], which focus its attention of comparing the spectra of two overlapping peak clusters to obtain the pure spectrum of the common component in the two overlapping peaks and further to resolve the whole overlapping peak cluster. With the help of SFA, six common components were extracted from the two peak clusters, assigned B1-B6 in the sample from extraction method 1 and b1-b6 in the sample from extraction method II (Fig. 10 (medium part) and (bottom part), respectively). For more detail, the reader can consult reference [202].

Table 2

Comparison of correlative components and their chromatographic eluting time (min) of Ginkgo biloba sample $\boldsymbol{X}_{1}$ with those of $\boldsymbol{X}_{2}$

\begin{tabular}{llllcccr}
\hline $\begin{array}{l}\text { Correlation } \\
\text { component no. }\end{array}$ & $\begin{array}{l}\text { Retention time } \\
\left(t_{1, k}\right) \text { of } \boldsymbol{X}_{1}\end{array}$ & $\begin{array}{l}\text { Retention time } \\
\left(t_{2, k}\right) \text { of } \boldsymbol{X}_{2}\end{array}$ & $\begin{array}{l}\Delta t=t_{1, k}-t_{2, k} \\
(\mathrm{~min})\end{array}$ & $\begin{array}{l}\text { Correlation } \\
\text { component no. }\end{array}$ & $\begin{array}{l}\text { Retention time } \\
\left(t_{1, k}\right) \text { of } \boldsymbol{X}_{1}\end{array}$ & $\begin{array}{l}\text { Retention time } \\
\left(t_{2, k}\right) \text { of } \boldsymbol{X}_{2}\end{array}$ & $\begin{array}{l}\Delta t=t_{1, k}-t_{2, k} \\
(\mathrm{~min})\end{array}$ \\
\hline 1 & 2.0820 & 1.7020 & 0.3800 & 7 & 9.4220 & 8.3353 & 1.0867 \\
2 & 2.5287 & 2.1153 & 0.4134 & 8 & 10.3487 & 9.2553 & 1.0934 \\
3 & 2.9953 & 2.4753 & 0.5200 & 9 & 10.8887 & 9.7287 & 1.1600 \\
4 & 5.5287 & 4.7153 & 0.8134 & 10 & 11.2820 & 10.0287 & 1.2533 \\
5 & 6.9953 & 6.1020 & 0.8933 & 11 & 15.7020 & 14.6420 & 1.0600 \\
6 & 8.2620 & 7.2087 & 1.0533 & 12 & 18.7620 & 17.6020 & 1.1600 \\
\hline
\end{tabular}



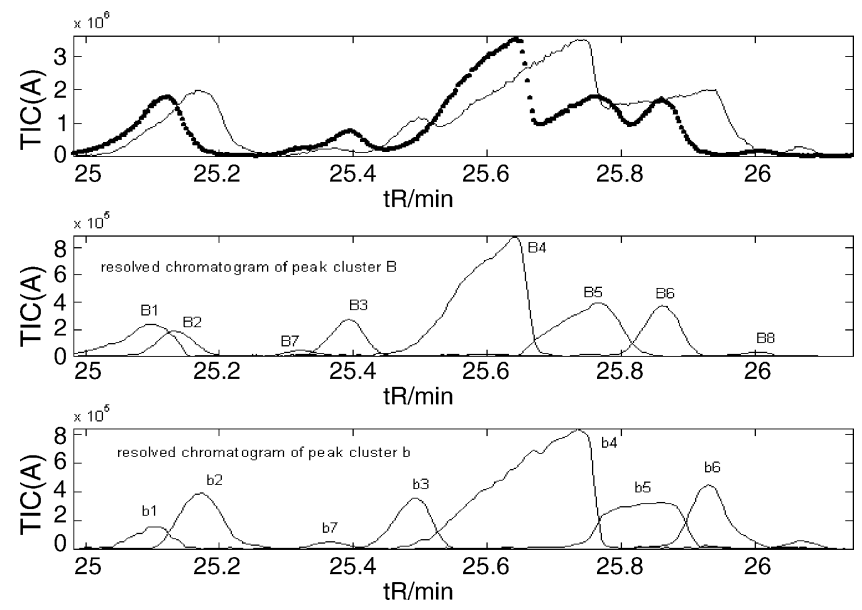

Fig. 10. Total ion current chromatograms of (top part) peak clusters B (bold dotted line) and $b$ (thin continuous line) as marked in Fig. 9 and (medium part) and (bottom part) their corresponding resolved chromatograms, respectively.

In fact, there are lots of chemometric methods [203-215] developed recently to deal with the overlapping chromatographic peaks with the help of the two-way data from hyphenated chromatography. For more detail, the readers can refer to a review paper by one of authors of this review and the references therein [198].

\section{Further comments on quality control of herbal medicines}

Western and traditional Chinese medical practices represent totally different philosophies [227,228]. Thus, this is not a simple exercise of applying modern technologies to quality control of the products that have been in constant use for centuries. The progress on quality control of herbal medicines discussed in this review is just at its beginning stage of a long journey.

Of course, the proposal of the use of chromatographic fingerprints of herbal medicines for quality control of herbal medicines is definitely a progress. However, using the chemical fingerprints for the purpose of quality control of herbal medicines can only address to the problem of comparing the integrated sameness and/or difference and controlling their stability of the available herbal products. The complex relationship between the chromatographic fingerprints and efficacy of the herbal medicines (QRFE) is not taken into account yet, which seems to be the most important aspect for the quality control of herbal medicines. As it is well known that the efficacy of traditional herbal medicines has a characteristic of a complex mixture of chemical compounds present in the herbs, thus how to evaluate reasonably their relationship is obviously not a trivial task. THMs represent a much more daunting challenge due to the natural variability of the individual herbs and the chemical complexity of the formulations. Moreover, the chemical profile by itself is insufficient in determining the efficacy of TCM [226]. This is where biochemistry, molecular biology, and cell biology are invaluable in establishing quantifiable and reproducible assays [216]. Chemical fingerprints might be linked to these biological assays to provide assurance of efficacy and consistency. But the research work on this aspect, to our best knowledge, is far from sufficient to meet the criteria needed.

Thus, the researches concerning the relationship between the chromatographic fingerprints and efficacy of the herbal medicines are urgent requirements for the quality control of herbal medicines. On the other hand, the works on possible contaminations in herbal products, such as excessive or banned pesticides, microbial contaminants, heavy metals, chemical toxins, should be also conducted concurrently [217]. In fact, the research field of quality control of herbal medicines is really an interdisciplinary research. It needs crossover of chemistry, pharmacology, medicine and even statistics to provide a platform for the quality control of traditional herbal medicines and further to discover the novel therapeutics composed of multiple chemical compounds.

\section{Acknowledgement}

The financial support from the National Natural Science Foundation of the People's Republic of China (grant nos. 20175036 and 20235020) are greatly appreciated.

\section{References}

[1] WHO, General Guidelines for Methodologies on Research and Evaluation of Traditional Medicines, 2000, p. 1.

[2] X.J. Yan, J.J. Zhou, G.R. Xie, G.W.A. Milne, Traditional Chinese Medicines: Molecular Structures, Natural Sources and Applications, Aldershot, Ashgate, 1999.

[3] P.H. Raven, R.F. Evert, S.E. Eichhorn, Biology of Plants, sixth ed., Freeman, New York, 1999.

[4] R. Bauer, Drug Inform. J. 32 (1998) 101.

[5] V.E. Tyler, J. Nat. Prod. 62 (1999) 1589.

[6] State Drug Administration of China, Chin. Tradit. Pat. Med. 22 (2000) 671.

[7] E.S. Ong, J. Sep. Sci. 25 (2002) 825.

[8] FDA Guidance for Industry-Botanical Drug Products (Draft Guidance), 2000, VIII, B, 2e; 3e.

[9] R. Upton, International Symposium on Quality of Traditional Chinese Medicine with Chromatographic Fingerprint, Guangzhou, 2001, i 2-1.

[10] J.D. Philipsom, British Herbal Pharmacopoeia, British Herbal Medicine Association Publications, 1996. Forward.

[11] P.S. Xie, Tradit. Chin. Drug Res. Clin. Pharm. 12 (2001) 141.

[12] L. Kelly, International Symposium on Quality of Traditional Chinese medicine with Chromatographic Fingerprint, Guangzhou, 2001, i 4-1.

[13] W.J. Welsh, W.K. Lin, S.H. Tersigni, E. Collantes, R. Duta, M.S. Carey, W.L. Zielinski, J. Brower, J.A. Spencer, T.P. Layloff, Anal. Chem. 68 (1996) 3473.

[14] P. Valentão, P.B. Andrade, F. Areias, F. Ferreres, R.M. Seabra, J. Agric. Food Chem. 47 (1999) 4579.

[15] N.J. Lazarowych, P. Pekos, Drug Inform. J. 32 (1998) 497.

[16] F. Gong, Y.Z. Liang, H. Cui, F.T. Chau, B.T.P. Chau, J. Chromatogr. A 909 (2001) 237. 
[17] F. Gong, Y.Z. Liang, Q.S. Xu, F.T. Chau, A.K.M. Leung, J. Chromatogr. A 905 (2001) 193.

[18] F. Gong, Y.Z. Liang, Q.S. Xu, F.T. Chau, K.M. Ng, Anal. Chim. Acta 450 (2001) 99.

[19] K.S. Booksh, B.R. Kowalski, Anal. Chem. 66 (1994).

[20] A. Hasler, O. Sticher, J. Chromatogr. 605 (1992) 41.

[21] O. Sticher, Planta Med. 59 (1993) 2.

[22] E. Revilla, E.G. Beneytez, F. Cabello, G.M. Ortega, J.M. Ryan, J. Chromatogr. A 915 (2001) 53.

[23] R.M. Smith, M.D. Burford, Chemom. Intell. Lab. Syst. 18 (1993) 285.

[24] D.J. Zeeb, B.C. Nelson, K. Albert, J.J. Dalluge, Anal. Chem. 72 (2000) 5020.

[25] C. Peres, C. Viallon, J.L. Berdague, Anal. Chem. 73 (2001) 1030.

[26] J. Faghihi, X.F. Jiang, R.V.S. Goldman, S. Sharfstein, J. Sarver, P. Erhardt, J. Chromatogr. A 915 (2001) 61.

[27] F.T. Chau, S.K. Tsui, S.K. Wong, F. Gong, B.T.P. Chan, L.Q. Huang, Y.Z. Liang, Tradit. Chin. Drug Res. Clin. Pharm. 12 (2001) 164.

[28] Y.Z. Liang, Tradit. Chin. Drug Res. Clin. Pharm. 12 (2001) 196.

[29] K. Heinig, C. Voht, G. Werner, Anal. Chem. 70 (1998) 1885.

[30] G.L. Aloonso, M.R. Salinas, J. Garijo, J. Food Prot. 61 (1998) 1525.

[31] C. Gherman, M. Culea, O. Cozar, Talanta 53 (2000) 253.

[32] M. Rajani, M.N. Ravishankara, N. Shrivastava, H. Padh, J. Planar Chromatogr. 14 (2001) 34.

[33] F.M. Areias, P. Valentão, P.B. Andrade, F. Ferreres, R.M. Seabra, Food. Chem. 73 (2000) 307.

[34] X.G. He, L.Z. Lin, L.Z. Lian, M. Lindenmaier, J. Chromatogr. A 818 (1998) 127.

[35] A. Rehwald, B. Meier, O. Sticher, J. Chromatogr. A 677 (1994) 25.

[36] X.G. He, L.Z. Lin, M.W. Bernart, L.Z. Lian, M. Lindenmaier, J. Chromatogr. A 815 (1998) 205.

[37] K. Krizsán, G. Szókán, Z.A. Tóth, F. Hollósy, M. László, A. Khlafulla, J. Liq. Chromatogr. Rel. Technol. 19 (1996) 2295.

[38] D.W. Choi, J.H. Kim, S.Y. Cho, D.H. Kim, S.Y. Chang, Toxicology $181 / 182$ (2002) 581

[39] H. Wagner, S. Bladt, V. Rickl, Plant Drug Analysis: A Thin Layer Chromatography Atlas, second ed., Springer-Verlag, 1996.

[40] A. Baerheim Svendsen, J. Planar Chromatogr. Modern TLC 2 (1989) 8.

[41] G.M. Monfort, T.T. Ayora, M. Maldonado, I.V. Loyhola, Phytochem. Anal. 3 (1992) 117.

[42] W. Funk, B. droeschel, J. Planar Chromatogr. Modern TLC 4 (1991) 123

[43] M. Govindan, G. Govindan, Fitoterapia 71 (2000) 232.

[44] I. Barene, I. Daberte, L. Zvirgzdina, V. Iriste, Medicina 39 (2003) 127.

[45] M.A. King-Wah, C.H.A.U. Foo-Tim, W.U. Jian-Yong, Chin. J. Chem. 22 (2004) 85.

[46] G. Matysik, H. Giryn, Chromatographia 42 (1996) 555.

[47] G. Matysik, Chromatographia 43 (1996) 39.

[48] W. Markowski, E. Soczewinski, Chromatographia 36 (1993) 330.

[49] C.F. Poole, M.T. Belay, J. Planar Chromatogr. Modern TLC 4 (1991) 345 .

[50] B. Simonovska, I. Vovk, S. Andrenšek, K. Valentová, J. Ulrichová, J. Chromatogr. A 1016 (2003) 89.

[51] F.T. Chau, T.P. Chan, J. Wang, Bioinformatics (formerly Comput. Appl. Biosci.) 14 (1998) 540.

[52] S. Nyiredy, J. Chromatogr. A 1000 (2003) 985.

[53] R. Brochmann-Hanssen, A. Baerheim Svendsen, J. Pharm. Sci. 51 (1962) 1095.

[54] P. Majlat, J. Chromatogr. 241 (1982) 399.

[55] C.J. Briggs, K.J. Simons, J. Chromatogr. 257 (1983) 132

[56] P. Majlat, Pharmazie 39 (1984) 325.
[57] G.J. Soleas, E.P. Diamandis, A. Karumanchiri, D.M. Goldberg, Anal. Chem. 69 (1997) 4405.

[58] R.J. Molyneux, N. Mahoney, P. Bayman, R.Y. Wong, K. Meyer, N. Irelan, J. Agric. Food Chem. 50 (2002) 1393.

[59] F. Angerosa, N. d'Alessandro, F. Corana, G. Mellerio, J. Chromatogr. A 736 (1996) 195.

[60] M. Bunzel, J. Ralph, J.M. Marita, R.D. Hatfield, H. Steinhart, J. Sci. Food Agric. 81 (2001) 653.

[61] A. El-Shazly, A. Tei, L. Witte, M. El-Domiaty, M. Wink, Z. Naturforsch. C. J. Biosci. 52 (1997) 729.

[62] M. Ylinen, T. Naaranlahti, S. Lapinjoki, A. Huhtikangas, M.L. Salonen, L.K. Simola, M. Lounasmaa, Planta Med. 52 (1986) 85.

[63] N. Li, G. Lin, Y.-W. Kwan, Z.-D. Min, J. Chromatogr. A 849 (1999) 349.

[64] C.-L. Liu, P.-L. Zhu, M.-C. Liu, J. Chromatogr. A 857 (1999) 167

[65] G. Lin, P. Li, S.L. Li, S.W. Chan, J. Chromatogr. A 935 (2001) 321.

[66] T.R. Tsai, T.Y. Tseng, C.F. Chen, T.H. Tsai, J. Chromatogr. A 961 (2002) 83.

[67] Kitagawa, T.Y. I., Pure Appl. Chem. 74 (2002) 1189.

[68] U. Sanyal, S. Bhattacharyy, A. Patra, B. Hazra, J. Chromatogr. A 1017 (2003) 225

[69] C. Thanawiroon, R.J. Linhardt, J. Chromatogr. A 1014 (2003) 215.

[70] D. Loganathan, H.M. Wang, L.M. Mallis, R.J. Linhardt, Biochemistry 29 (1990) 4362

[71] U.R. Desai, H.M. Wang, T.R. Kelly, R.J. Linhardt, Carbohydr. Res. 241 (1993) 135.

[72] T. Imanari, T. Toida, I. Koshiishi, H. Toyoda, J. Chromatogr. A 720 (1996) 275.

[73] S. Leonard, R. Capote, N. Germonprez, L. Van Puyvelde, N. De Kimpe, H. Vermeersch, J. Rosier, L. Maes, E. Roets, J. Hoogmartens, J. Chromatogr. A 1012 (2003) 39.

[74] C.H. Lin, B.H. Chen, J. Chromatogr. A 1012 (2003) 103.

[75] G.R. Cawthray, J. Chromatogr. A 1011 (2003) 233.

[76] K. Albert, W.M. Lee, W.F. Chan, Y.Z. Liang, K.T. Fang, Chemom. Intell. Lab. Syst. 39 (1997) 11.

[77] K. Sayler, R. Weinberger, J. Chromatogr. A 1014 (2003) 179.

[78] A. Pervin, C. Gallo, K.A. Jandik, X.J. Han, R.J. Linhardt, Glycobiology 5 (1995) 83 .

[79] D. Loganathan, H.M. Wang, L.M. Mallis, R.J. Linhardt, Biochemistry 29 (1990) 4362

[80] N.K. Karamanos, P. Vanky, G.N. Tzanakakis, T. Tsegenidis, A. Hjerpe, J. Chromatogr. A 765 (1997) 169.

[81] K.G. Rice, R.J. Linhardt, Carbohydr. Res. 190 (1989) 219.

[82] R. Niemi, H. Taipale, M. Ahlmark, J. Chromatogr. B 701 (1997) 97.

[83] W.W. Christie, in: W.W. Christie (Ed.), Advances in Liquid Methodology, vol. 1, Oily Press, 1992, p. 257.

[84] P. Nebinger, M. Koel, A. Franz, E. Werries, J. Chromatogr. 265 (1983) 19.

[85] J.W. Jorgensen, K.D. Lukacs, Anal. Chem. 53 (1981) 1298.

[86] J.J. Yang, H. Long, H.W. Liu, A.J. Huang, Y.L. Sun, J. Chromatogr. A 811 (1998) 274.

[87] K. Gunaydýn, F.B. Erim, J. Chromatogr. A 954 (2002) 291.

[88] G. Chen, H. Zhang, J. Ye, Anal. Chim. Acta 423 (2000) 69

[89] Y.M. Liu, S.J. Sheu, S.H. Chiou, S.H. Chang, Y.P. Chen, Planta Med. 59 (1993) 376.

[90] Y.M. Liu, S.J. Sheu, S.H. Chiou, S.H. Chang, Y.P. Chen, Phytochem. Anal. 5 (1994) 256.

[91] W.C. Chuang, S.K. Wu, S.J. Sheu, S.H. Chiou, H.C. Chang, Y.P. Chen, Planta Med. 61 (1995) 459.

[92] W.C. Chuang, S.K. Wu, S.J. Sheu, S.H. Chiou, H.C. Chang, Y.P. Chen, Planta Med. 61 (1996) 459.

[93] Yang, J., Long, H., Liu, Y.P. H., J. Huang, L. A., E. Sun, J. Y., J. Chromatogr. A 811 (1998) 274.

[94] H. Stuppner, M. Ganzera, J. Chromatogr. A 717 (1995) 271.

[95] S.S. Yang, I. Smetena, Chromatographia 40 (1995) 375. 
[96] Y.M. Liu, S.J. Sheu, J. Chromatogr. 623 (1992) 196.

[97] Y.M. Liu, S.J. Sheu, J. Chromatogr. 639 (1993) 323.

[98] Y.M. Liu, S.J. Sheu, J. Chromatogr. 634 (1993) 329.

[99] H. Stuppner, S. Sturm, G. Konwalinka, J. Chromatogr. 609 (1992) 375.

[100] V.C. Trenerry, R.J. Wells, J. Robertson, J. Chromatogr. A 718 (1995) 217.

[101] H. Stuppner, S. Sturm, N. Mulinacci, F. Vincieri, Chromatographia 37 (1993) 579.

[102] P. Pietta, P. Mauri, A. Rava, G. Sabbatini, J. Chromatogr. 549 (1991) 367.

[103] U. Seitz, G. Bonn, P. Oefner, M. Popp, J. Chromatogr. 559 (1991) 499.

[104] P. Pietta, A. Bruno, P. Mauri, A. Rava, J. Chromatogr. 593 (1992) 165.

[105] T.K. McGhie, J. Chromatogr. 634 (1993) 107.

[106] P. Pietta, P. Mauri, A. Bruno, L. Zini, J. Chromatogr. 638 (1993) 357.

[107] Z.K. Shihabi, T. Kute, L.L. Garcia, M. Hinsdale, J. Chromatogr. A 680 (1994) 181.

[108] Y.-Y. Zong, M.-T. Jin, Yaoxue Xuebao 30 (1995) 594.

[109] Z.K. Shibabi, M.E. Hinsdale, Electrophoresis 16 (1995) 2159.

[110] E. Dose, G. Guiochon, Anal. Chem. 63 (1991) 1154.

[111] G. Guetens, G. De Boeck, M. Wood, R.A.A. Maes, A.A.M. Eggermont, M.S. Highley, A.T. van Oosterom, E.A. de Bruijn, U.R. Tjaden, J. Chromatogr. A 976 (2002) 229.

[112] F. Gong, Y.Q. Song, Y.G. Peng, Y.Z. Liang, A.K.M. Leung, F.T. Chau, Acta Pharm. Sin. 35 (2000) 394 (in Chinese).

[113] F. Gong, Y. Liang, A.K.M. Leung, F.T. Chau, Chin. Anal. Chem. 28 (2000) 860 (in Chinese).

[114] Fan Gong, Y.Z. Liang, C. Hui, F.T. Chau, B.T.P. Chan, J. Chromatogr. A 905 (2001) 193.

[115] F. Gong, Y.Z. Liang, Q.S. Xu, F.T. Chau, J. Chromatogr. A 909 (2001) 237.

[116] X.N. Li, H. Cui, Y.Q. Song, Y.Z. Liang, Acta Pharm. Sin. 36 (2001) 215 (in Chinese).

[117] F. Gong, Y.Z. Liang, Y.Q. Song, Y.G. Peng, H. Cui, A.K.M. Leung, F.T. Chau, Chem. J. Chin. Univ. 22 (2001) 1481 (in Chinese).

[118] X.N. Li, Y.Z. Liang, Phytochem. Anal. 14 (2003) 23.

[119] F. Gong, Y.Z. Liang, J. Sep. Sci. 26 (2003) 112.

[120] B.Y. Li, Y.Z. Liang, Chromatogrphia 57 (2003) 235.

[121] X.S. Wang, V. Kapoor, G.A. Smythe, Am. J. Chin. Med. 31 (2003) 927.

[122] P.P. Fu, Y.C. Yang1, Q.S. Xia, M.W. Chou, Y.Y. Cui, G. Lin, J. Food Drug Anal. 10 (2002) 198.

[123] M.C. Tseng, M.J. Tsai, J.H. Lin, K.C.H. Wen, J. Food Drug Anal. 8 (2000) 315.

[124] R.F. Vieira, J.E. Simon, Econ. Bot. 54 (2002) 207.

[125] C. Gherman, M. Culea, O. Cozar, Talanta 53 (2000) 253.

[126] A. Velasco-Negueruela, M.J. Perez-Alonso, P.L. Perez de Paz, J. Pala-Paul, J. Sanz, J. Chromatogr. A 984 (2003) 159.

[127] A. Velasco-Negueruela, M.J. Perez-Alonso, P.L. Perez de Paz, J. Pala-Paul, J. Sanz, J. Chromatogr. A 1011 (2003) 241.

[128] F.A. Mellon, J.R. Chapman, J.A.E. Pratt, J. Chromatogr. 394 (1987) 209.

[129] M.P. Maillard, J.L. Wolfender, K. Hostettmann, J. Chromatogr. 647 (1993) 147.

[130] J.L. Wolfender, M.P. Maillard, K. Hostettmann, J. Chromatogr. 647 (1993) 183.

[131] J.L. Wolfender, M.P. Maillard, K. Hostettmann, Phytochem. Anal. 5 (1994) 153.

[132] J.L. Wolfender, K. Hostettmann, in: J.T. Arnason, R. Mata, J.T. Romeo (Eds.), Phytochemistry of Medicinal Plants, Recent Advances in Phytochemistry, vol. 29, Plenum Press, New York, 1995, p. 189.

[133] S. Zhou, M. Hamburger, J. Chromatogr. A 755 (1996) 189.
[134] J.L. Wolfender, S. Rodriguez, K. Hostettmann, J. Chromatogr. A 794 (1998) 299.

[135] M. Careri, A. Mangia, M. Musci, J. Chromatogr. A 794 (1998) 263.

[136] Hayen, H., M. Karst, J. U., J. Chromatogr. A 1000 (2003) 549.

[137] G.C. Kite, M.A. Yule, C.J. Leon, M.S.J. Simmonds, Rapid Commun. Mass Spectrom. 16 (6) (2002) 585.

[138] G.C. Kite, M.J.R. Howes, C.J. Leon, M.S.J. Simmonds, Rapid Commun. Mass Spectrom. 17 (3) (2003) 238.

[139] G. Guetens, G. De Boeck, M. Wood, R.A.A. Maes, A.A.M. Eggermont, M.S. Highley, A.T. van Oosterom, E.A. de Bruijn, U.R. Tjaden, J. Chromatogr. A 976 (2002) 239.

[140] J.-L. Wolfender, K. Ndjoko, K. Hostettmann, Phytochem. Anal. 12 (2001) 2.

[141] K. Albert, J. Chromatogr. A 856 (1999) 199.

[142] W.M. Niessen, J. Chromatogr. A 856 (1999) 179.

[143] W.M.A. Niessen, Liq. Chromatogr. Mass Spectrom., second ed., Dekker, New York, 1999.

[144] T. Visser, M.J. Vredenbergregt, G.J. ten Hove, A.P.J.M. de Jong, G.W. Somsen, Anal. Chim. Acta 342 (1997) 151

[145] G. Bringmann, K. Messer, M. Wohlarth, J. Kraus, K. Dumbuya, M. Ruckert, Anal. Chem. 71 (1999) 2678.

[146] J.-L. Wolfender, S. Rodriguez, K. Hostettmann, J. Chromatogr. A 794 (1998) 299.

[147] B. Drager, J. Chromatogr. A (2002) 978.

[148] K. Robards, J. Chromatogr. A 1000 (2003) 657.

[149] X.-G. He, J. Chromatogr. A 880 (2000) 203.

[150] E.M. Lenz, D. Greatbanks, I.D. Wilson, M. Spraul, M. Hofmann, J. Troke, J.C. Lindon, J.K. Nicholson, Anal. Chem. 68 (1996) 2832.

[151] J. Chin, J.B. Fell, M. Jarosinski, M.J. Shapiro, J.R. Wareing, J. Org. Chem. 63 (1998) 386.

[152] J.C. Lindon, R.D. Farrant, P.N. Sanderson, P.M. Doyle, S.L. Gough, M. Spraul, M. Hofmann, J.K. Nicholson, Magn. Reson. Chem. 33 (1995) 857.

[153] M.E. Lacey, Z.J. Tan, A.G. Webb, J.V. Sweedler, J. Chromatogr. A 922 (2001) 139.

[154] M. Godejohann, A. Preiss, C. Mugge, G. Wunsch, Anal. Chem. 69 (1997) 3832.

[155] J.L. Wolfender, K. Ndjoko, K. Hostettmann, Phytochem. Anal. 12 (2001) 2.

[156] D. Louden, A. Handley, S. Taylor, E. Lenz, S. Miller, I.D. Wilson, A. Sage, R. Lafont, J. Chromatogr. A 910 (2001).

[157] J. Wittig, S. Wittemer, M. Veit, J. Chromatogr. B 761 (2001) 125.

[158] D.J. Jones, C.K. Lim, D.R. Ferry, A. Gescher, Biomed. Chromatogr. 12 (1998) 232.

[159] P.H. Gamache, I.N. Acworth, Proc. Soc. Exp. Biol. Med. 217 (1992).

[160] R. Bugianesi, M. Serafini, F. Simone, D. Wu, S. Meydani, A. FerroLuzzi, E. Azzini, G. Maiani, Anal. Biochem. 284 (2000) 296.

[161] H.T. Feng, L.L. Yuan, S.F.Y. Li, J. Chromatogr. A 1014 (2003) 83.

[162] S. Strum, H. Stuppner, Electrophoresis 19 (1998) 3026.

[163] F.Y.L. Hsieh, J.Y. Cai, J. Henion, J. Chromatogr. A 679 (1994) 206.

[164] M. Unger, D. Stockigt, D. Belder, J. Stockigt, J. Chromatogr. A 767 (1997) 263

[165] J. Stockigt, Y. Sheludko, M. Unger, I. Gerasimenko, H. Warzecha, D. Stockigt, J. Chromatogr. A 967 (2002) 85.

[166] K. Pusecker, J. Schewitz, P. Gfrorer, L.H. Tseng, K. Albert, E. Bayer, I.D. Wilson, N.J. Bailey, G.B. Scarfe, J.K. Nicholson, J.C. Lindon, Anal. Commun. 35 (1998) 3159.

[167] J. Schewitz, P. Gfrorer, K. Pusecker, L.H. Tseng, K. Albert, E. Bayer, I.D. Wilson, N.J. Bailey, G.B. Scarfe, J.K. Nicholson, J.C. Lindon, Analyst 123 (1998) 2835.

[168] Y. Hayashi, R. Matsuda, A. Nakamura, J. Chromatogr. Sci. 28 (1990) 628.

[169] R. Matsuda, Y. Hayashi, M. Ishibashi, Y. Takeda, J. Chromatogr. 462 (1989) 13. 
[170] R. Matsuda, Y. Hayashi, M. Ishibashi, Y. Takeda, J. Chromatogr. 462 (1989) 23.

[171] J.F.K. Huber, E. Kenndler, G. Reich, W. Hack, J. Wolf. Anal. Chem. 65 (1993) 2903.

[172] R. Matsuda, Y. Hayashi, M. Ishibashi, Y. Takeda, J. AOAC Int. 77 (1994) 338.

[173] F. Gong, Y.Z. Liang, P.S. Xie, A.J. Sung, J. Chromatogr. A 1002 (2003) 25.

[174] Y.Q. Ru, Information-Theoretical Fundamentals of Modern Analytical Chemistry, Hunan University press, Changsha, PR China, 2004 (in Chinese).

[175] J. Calvin Giddings, Unified Separation Science, Wiley, New York, 1991.

[176] N.P.V. Nielsen, J.M. Carstensen, J. Smedsgarrd, J. Chromatogr. A 805 (1998) 17.

[177] J.A. Pino, J.E. McMurry, P.C. Jurs, B.K. Lavine, A.M. Harper, Anal. Chem. 57 (1985) 295.

[178] M.E. Parrish, B.W. Good, F.S. Hsu, F.W. Hatch, D.M. Ennis, D.R. Douglas, J.H. Shelton, D.C. Watson, Anal. Chem. 53 (1981) 826.

[179] K.J. Johnson, B.W. Wright, K.H. Jarman, R.E. Synovec, J. Chromatogr. A 996 (2003) 141.

[180] G. Malmquist, R. Danielsson, J. Chromatogr. A 687 (1994) 71.

[181] D. Bylund, R. Danielsson, G. Malmquist, K.E. Markides, J. Chromatogr. A 961 (2002) 237.

[182] M.D. Hämäläinen, Y.Z. Liang, O.M. Kvalheim, R. Andersson, Anal. Chim. Acta 271 (1993) 101.

[183] F. Gong, Y.Z. Liang, Y.S. Fung, J. Chromatogr. A 1029 (2004) 173.

[184] S. Wold, K. Esbensen, P. Geladi, Chemom. Intell. Lab. Syst. 2 (1987) 37

[185] H. Martens, T. Naes, Multivariate Calibration, second ed., Wiley, New York, 1991.

[186] M.H. Zhang, Q.S. Xu, D.L. Massart, Chemom. Intell. Lab. Syst. 67 (2003) 175.

[187] J. Zupan, S. Bohance, M. Razinger, et al., Anal. Chim. Acta 210 (1988) 63.

[188] B. Walczak, D.L. Massart, Trends Anal. Chem. 16 (1997) 451.

[189] F.T. Chau, T.M. Shih, J.B. Gao, et al., Appl. Spectrosc. 50 (1996) 339.

[190] W.J. Welsh, W. Lin, S.H. Tersigni, E. Collantes, R. Duta, M.S. Carey, Anal. Chem. 68 (1996) 3473.

[191] P.S. Xie, Tradit. Chin. Drug Res. Clin. Pharm. 12 (2001) 141.

[192] Y.Y. Cheng, M.J. Chen, W.D. Tong, J. Chem. Inform. Comput. Sci. 43 (2003) 1068.

[193] S. Wold, M. Sjostrom, in: B.R. Kowalski (Ed.), Chemometrics: Theory and Applications, ACS Ser., vol. 52, 1977, p. 243.

[194] E.R. Collantes, R. Duta, W.J. Welsh, W.L. Zielinski, J. Brower, Anal. Chem. 69 (1997) 1392.

[195] Y.Y. Cheng, M.J. Chen, W.J. Welsh, J. Chem. Inform. Comput. Sci. (2003) 43.

[196] F. Vogt, B. Mizaikoff, J. Chemom. 17 (2003) 225.
[197] F. Vogt, B. Mizaikoff, Anal. Chem. 75 (2003) 3050.

[198] Y.Z. Liang, O.M. Kvalheim, Fresenius J. Anal. Chem. 370 (2001) 694.

[199] B.Y. Li, Y. Hu, Y.Z. Liang, J. Sep. Sci., in press.

[200] Y. Hu, Y.Z. Liang, B.Y. Li, C.J. Xu, Z.D. Zeng, Acta Chim. Sin. 61 (2003) 1466.

[201] R. Manne, H.L. Shen, Y.Z. Liang, Chemo. Intell. Lab. Sys. 45 (1999) 171.

[202] X.N. Li, H. Cui, Y.Q. Song, Y.Z. Liang, Phytochem. Anal. 14 (2003) 23.

[203] EJ. Karjalainen, U. Karjalainen, Chem. Intell. Lab. Syst. 14 (1992) 423.

[204] R. Tauler, Casassas E, Chem. Intell. Lab. Syst. 14 (1992) 305.

[205] R. Tauler, A. Izquierdo-Ridorsa, E. Casassas, Chem. Intell. Lab. Syst. 18 (1992) 293.

[206] W. Windig, Chem. Intell. Lab. Syst. 36 (1997) 3.

[207] P.J. Gemperline, J. Chem. Inform. Comput. Sci. 24 (1984) 206.

[208] B.G.M. Vandeginste, T. Esser, T. Bosman, J. Reijnen, G. Kateman, Anal. Chem. 57 (1985) 971.

[209] M. Maeder, Anal. Chem. 59 (1987) 527.

[210] H.R. Keller, D.L. Massart, Chem. Intell. Lab. Syst. 12 (1992) 209.

[211] O.M. Kvalheim, Y.Z. Liang, Anal. Chem. 64 (1992) 936.

[212] Y.Z. Liang, O.M. Kvalheim, H.R. Keller, D.L. Massart, P. Kiechle, F. Erni, Anal. Chem. 64 (1992) 946.

[213] E.R. Malinowski, J. Chemom. 6 (1992) 29.

[214] Y.Z. Liang, O.M. Kvalheim, Anal. Chim. Acta 292 (1994) 5.

[215] E.R. Malinowski, Factor Analysis in Chemistry, second ed., Wiley, New York, 1991.

[216] R. Yuan, Y. Lin, Pharmacol. Ther. 86 (2000) 191.

[217] K. Chan, Chemosphere 52 (2003) 1361.

[218] F. Gong, Y.Z. Liang, Q.S. Xu, Anal. Chim. Acta 450 (2001) 99.

[219] B.Y. Li, Y. Hu, Y.Z. Liang, P.S. Xie, Y.P. Du, Anal. Chim. Acta (2004).

[220] B.Y. Li, Y.Z. Liang, Y. Hu, P.S. Xie, J. Chromatogr. A, submitted for publication.

[221] P.S. Xie, Y.Z. Yan, High Resolut. Chromatogr. Chromatogr. Commun. 10 (1987) 607.

[222] P.S. Xie, Y.Z. Yan, J. Planar Chromatogr. Modern TLC 1 (1988) 29.

[223] P.S. Xie, Y.Z. Yan, J. Planar Chromatogr. Modern TLC 1 (1988) 258.

[224] P.S. Xie, Y.Z. Yan, J. Planar Chromatogr. Modern TLC 5 (1992) 302.

[225] K. Chan, K.S. Bi, A.M. Wang, X. Luo, H.W. Yeung, Forensic Toxicology, Aristotle University, Thessaloniki, Greece, 1995.

[226] K. Chan, Anal. Sci. 17 (Suppl.) (2001) a409.

[227] L. Zhao, K. Chan, Med. Philos. 24 (2003) 58 (in Chinese).

[228] L. Zhao, K. Chan, Chin. J. Integr. Med. 9 (2003) 299-301.

[229] S.H. Chui, K. Chan, R. Wong, Meth. Find Exp. Clin. Pharmacol., in press. 